\title{
Computational Morphogenesis
}

Session Organizers: Makoto OHSAKI (Kyoto University), Hiroshi OHMORI (Nagoya University)

\section{Plenary Lecture: Abstract, Slides and Video}

Computational morphogenesis: Its current state and possibility for the future

Hiroshi OHMORI (Nagoya University)

\section{Keynote Lecture}

Linear mixed integer programming for topology optimization of trusses and plates

Makoto OHSAKI*, Ryo WATADA (Kyoto University)

Optimal design of glass grid shells with quadrilateral elements by means of a genetic algorithm

Mario SASSONE*, Alberto PUGNALE (Politecnico di Torino)

Development of intelligent truss optimization system

Seung-Chang LEE* , Jung-Keun OH (Samsung Corporation)

Shell surface with curved fold lines inspired by paper folding art

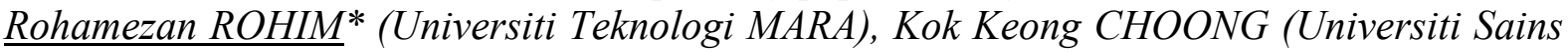
Malaysia), J. Y. KIM (Hyupsung University)

Modeling of clothing and interactions with the body using continuum degenerated shell finite elements

Colby C. SWAN*, Xiaolin MAN, Rob W. WILLIAMS (University of Iowa)

Structural analysis for multi-folding and deployable structures

Masatoshi NAKAZAWA (Tohoku Gakuin University), Ichiro ARIO* (Hiroshima University), Andrew WATSON (Loughborough University)

Structural behaviour of shell surface in the form of Möbius strip

$\underline{\text { Kok Keong CHOONG}}^{*}$, Min Sheng KUAN, (Universiti Sains Malaysia)

On the interaction between architecture and engineering: the acoustic optimization of a reinforced concrete shell

Mario SASSONE* (Politecnico di Torino), Tomàs MENDEZ (Caracas, Venezuela), Alberto PUGNALE (Politecnico di Torino)

Singularities

Peter MACAPIA* (Pratt Institute/Columbia University), Frank BITONTI, Robert BAKER, Charles KWAN (Pratt Institute)

Optimal structural shapes for shells using hybrid GA

Nidur SINGH, C.V. RAMAKRISHNAN*, D.K. SEHGAL (IIT Delhi)

For multiple-author papers:

Contact author designated by *

Presenting author designated by underscore 
Proceedings of the 6th International Conference on Computation of Shell and Spatial Structures

IASS-IACM 2008: "Spanning Nano to Mega"

28-31 May 2008, Cornell University, Ithaca, NY, USA John F. ABEL and J. Robert COOKE (eds.)

\title{
Computational morphogenesis: Its current state and possibility for the future
}

\author{
Hiroshi OHMORI* \\ *Nagoya University \\ Furo-cho Chikusa-ku Nagoya 464-8603, Japan \\ Email hero@dali.nuac.nagoya-u.ac.jp
}

\begin{abstract}
Computational morphogenesis is the word that is generally used for expressing those techniques or ways of thought by which the configuration or the system itself of the structures is generated mainly through the usage of the computers, which is realized on the firm foundation of both FEM as a tool of numerical analysis and various kinds of method based on relatively newly developed algorithms for structural optimization. Recently, it has been getting a considerable number of users such as structural engineers or engineering architects for the structural design of the actual buildings as well as the proposal for the architectural competitions. In this contribution, such state-of-the-art around the computational morphogenesis especially in Japan and the future prospect of the computational morphogenesis will be presented.
\end{abstract}

\section{Introduction}

From the viewpoint of structural design, it can be undoubtedly said that recent extraordinary rapid development of computers has been drastically changing their position at an unbelievably high speed. We already have had the simulation tools with which even highly complicated structural problems such as those containing geometrical as well as material nonlinearities should be simultaneously involved and, furthermore, even the cracking or the breaking of the materials should be correctly taken into account. Admirably precise computer simulations of the breaking process of reinforced concrete structures are good examples which clearly explain those situation. Moreover, it should be noted that truly innovative achievement in the field of structural topology optimization appeared in 1988, the fruit of which is called homogenization design method achieved by Bensoe and Kikuchi[1]. The author believes that it was really a breakthrough paving the way for utilization of structural optimization into the structural design, where methods based on the topology optimization are indispensable and essential. On the other hand, in almost the same period, Genetic Algorithms, one of the most efficient schemes for the arrangement optimization problem, has been developed and a lot of researches of the application of the algorithm have been started including those in the structural optimization. Genetic Algorithm has very distinctive characteristics such that it can deal with discrete variables with ease and, furthermore, plural solutions can be simultaneously obtained besides the best solution in the large. These characteristics of Genetic Algorithms are important when they are utilized as a tool of the structural design.

In this contribution, the present states of computational morphogenesis is surveyed where the fundamental methods such as truss topology optimization by Genetic Algorithms and both size and topology optimization by extended ESO (Evolutionary Structural Optimization) method are presented and, as the application of those methods, several projects, some of which are actually realized and the others proposed for the international competitions are shown. Moreover, methodology for the life cycle design (LCD) of structures is presented which are realized through the usage of Genetic Algorithms as the arrangement optimization tool in 4-D space, that is, the four dimension composed of three coordinates in physical space besides time. This is also a part of Computational Morphogenesis and the significance of LCD hereafter is expected to grow for the near future. 


\section{Computational Morphogenesis of Structures}

\subsection{Truss Structures}

Genetic Algorithm (GA) is one of the most effective optimization methods, which enables us to handle discrete variables. Owing to this characteristic, we can handle the standardized structural elements from which the optimal combination for the objective structures is investigated. Another advantage on using GA is that it gives pleural optimums beside the single best optimum solution, characteristic of which is very suitable for the process of structural design because the users or the designers can choose their preferable solution from those solutions proposed through the optimization process. Figure 1 shows the typical results for the truss dome and the truss roof structures, where the topologies as well as the combination of those structural elements are generated through the optimization process by GA (Kawamura et al. [3]).
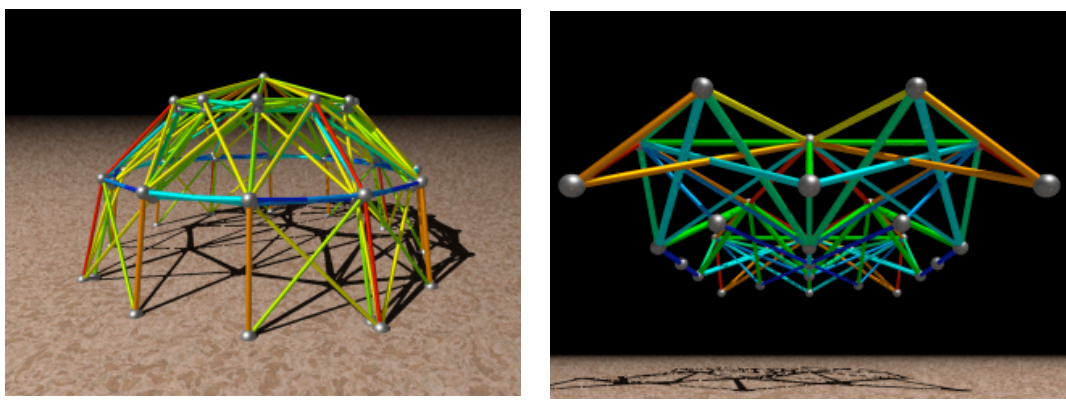

Figure 1: Truss Dome and Space Truss Structures Generated by GA

\subsection{Continuum Structures}

The author has proposed the Extended ESO (Evolutionary Structural Optimization) method by which we can obtain the solutions satisfying the constraint conditions required from the viewpoint of planning or other nonstructural requirements. The Extended ESO method has been developed through several modifications on the original ESO method which has had been proposed by Xie and Steven [5]. Figure 2 shows the result of the shell structure having the minimum volume subjected to the concentrated load at the apex, where the limit of the displacement in the vertical direction at the loading point is provided (Cui et al. [2]). As shown in the figure, we can see that pursuing the minimum volume structure, through the use of the Extended ESO method, generates the organic configuration.
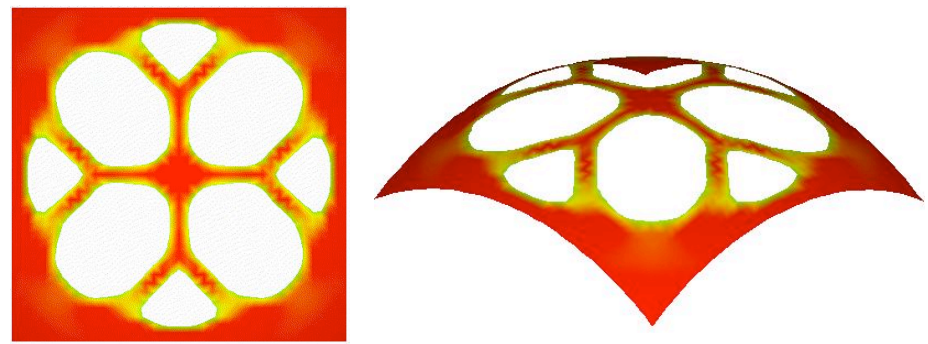

Figure 2: Shell Structure Generated by Extended ESO Method

The Extended ESO method which was originally developed and utilized for 2-D problems has been extended to the 3-D problems. In Figure 3, 3-D arch structure supporting the upper plate subjected to equally distributed load is shown, which has been generated through the 3-D extended ESO method.

On the other hand, the proposed Extended ESO method has been already applied to the structural design of the actual office building structure, the walls of which are designed based on the configuration generated by the extended ESO method as the initial design. Figure 4 shows the wall of the building as well as the intermediate process of the Extended ESO method (Ohmori et al. [4]), where we can see how the wall configuration has been changed through the modification process. 


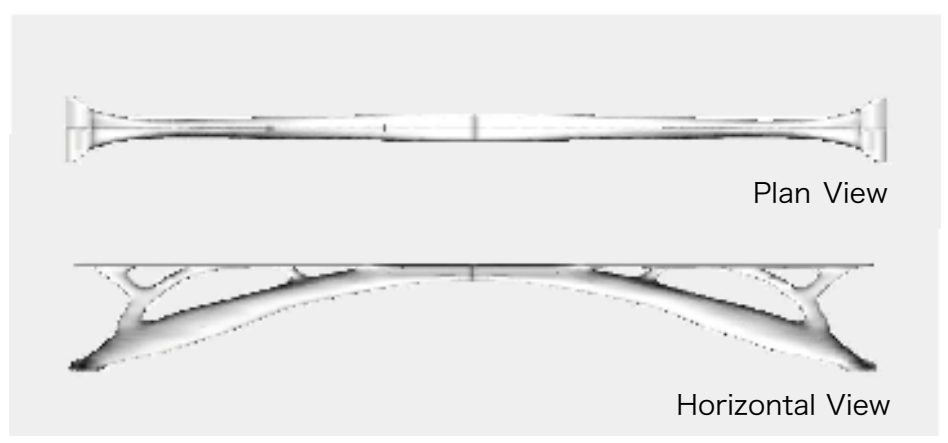

Figure 3: 3-D Arch Structure Generated by Extended ESO
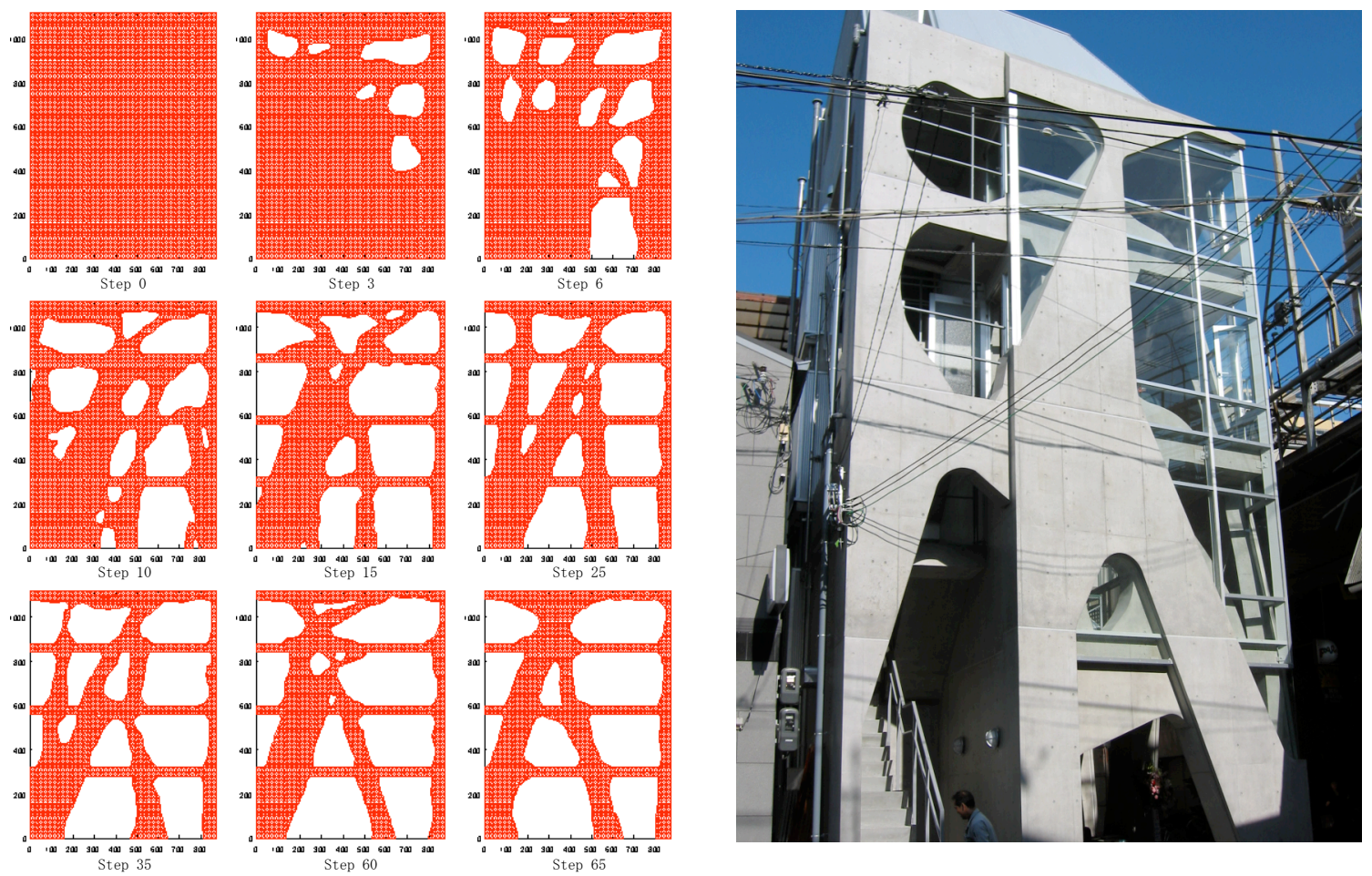

Figure 4: Structural Design of Wall through Extended ESO (Akutagawa Puroject)

\section{Life Cycle Structural Design}

It can be pointed out that most of conventional structures have been designed so that they can meet the requirements mainly coming from the initial performance from the viewpoint of their function, appearance as well as cost. However, the recent situation surrounding our environment has been drastically changing, that is, all industrial products should be produced as those whose impacts on the surrounding environment are reduced and controlled as possible as they can. Design of the structures is not an exception. Namely, we, structural engineers and designers, are requested to develop the design processes by which so-called sustainable structures are realized.

In this contribution, the authors propose the design method of the sustainable structures through the use of the optimization method by genetic algorithm, by which the optimal combinations of the structural elements as well as the optimal scenario of repair and preservation of the structures are obtained as the optimized solution (Figure 5). Generally, there are several objectives which have a trade-off relationship each other in life cycle design problems such as cost and structural performance. In those cases, the multi-objective optimization method can be effectively utilized and the multi-objective genetic algorithm is probably the most suitable technique for the 

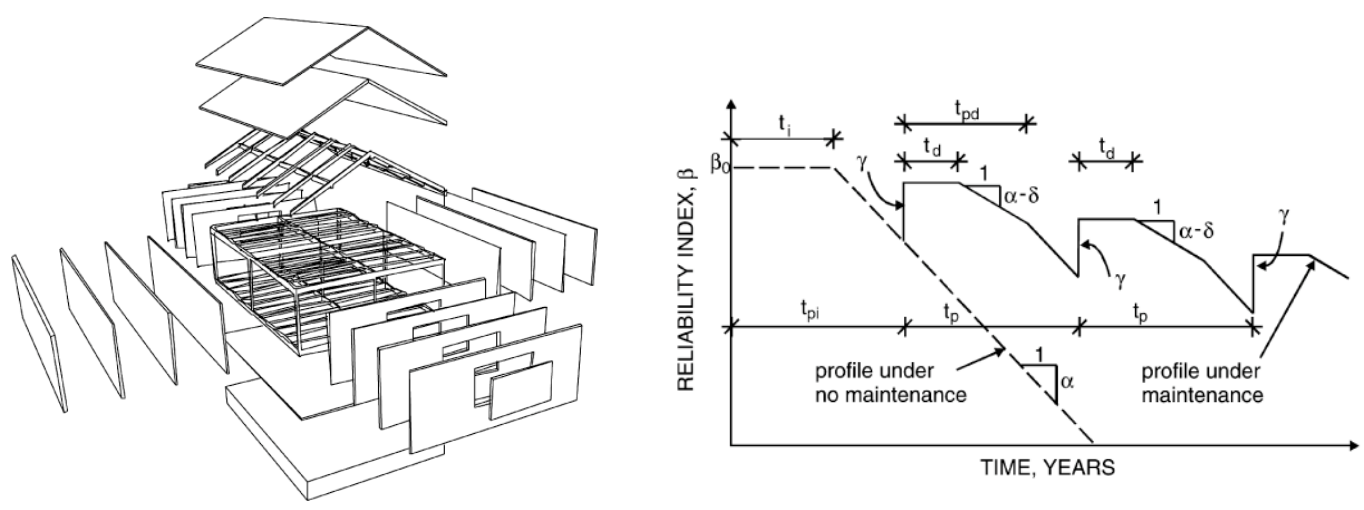

Figure 5: Life-Cycle Design of Structures

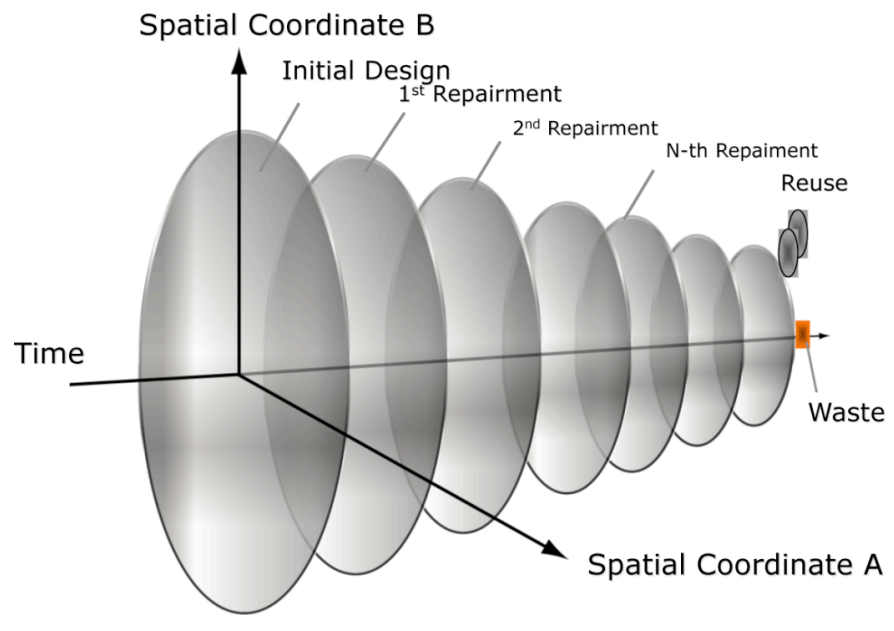

Figure 6: Arrangement Optimization in Time-Space Continuum

present problem. Figure 6 shows that the present multi-objective optimization problem toward the sustainable structures can be conceptually resulted in the optimization problem of the arrangement of the variables connected with the combination of structural elements as well as the time interval scheduling of the repair and preservation.

\section{References}

[1] Bensoe MP and Kikuchi N. Generating Optimal Topologies in Structural Design using a Homogenization Method, Computer Methods in Applied Mechanics and Engineering 1988; 71: 197-224.

[2] Cui C, Ohmori H and Sasaki M, Computational Morphogenesis of 3D Structures by Extended ESO Method, Journal of the International Association for Shell and Spatial Structures (IASS) 2003; 44(1): 51 61

[3] Kawamura H, Osada S and Ohmori H. Genetic Creation of 3-Dimensional Truss Structures, Journal of the International Association for Shell and Spatial Structures (IASS) 2000; 41(3): 163-175.

[4] Ohmori H, Futai H, Iijima T, Mutoh A and Hasegwa Y. Computational Morphogenesis and its Application to Structural Design, Proceedings of International Symposium on Shell and Spatial Structures, Theory, Technique, Valuation, Maintenance, Bucharest Poiana Brasov, Romania 2005, 13-20.

[5] Xie YM and Steven GP. Evolutionary Structural Optimization, Springer Verlag, 1997. 
Proceedings of the 6th International Conference on Computation of Shell and Spatial Structures

IASS-IACM 2008: "Spanning Nano to Mega"

\title{
Linear mixed integer programming for topology optimization of trusses and plates
}

\author{
Makoto OHSAKI*, Ryo WATADA \\ *Dept. of Architecture and Architectural engineering, Kyoto University \\ Kyotodaigaku Katsura, Nishikyo, Kyoto 615-8540, Japan \\ ohsaki@archi.kyoto-u.ac.jp
}

\begin{abstract}
A linear Mixed Integer Programming (MIP) approach is presented for topology optimization of trusses with discrete variables, and plates discretized to finite elements and subjected to in-plane loads. The problem with stress and displacement constraints is reformulated to linear MIP problem. The objective function is the total structural volume. It is shown in the numerical examples that an optimal combination of the traditional layouts of trusses can be successfully found by solving linear 0-1 MIP problems. An approximate substructure approach is developed for plates by consecutively optimizing the substructures, while the analysis is carried out for the whole structure. The effectiveness of the proposed method in view of computational cost is demonstrated in the numerical examples.
\end{abstract}

\section{Introduction}

Truss topology optimization problem is one of the traditional problems in structural optimization, and many methodologies have been developed (Bendsøe and Sigmund [1]). The most popular approach is the so called ground structure approach, where the cross-sectional areas are the continuous variables, and the unnecessary members are removed after optimization from the highly connected ground structure (Hagishita and Ohsaki [2]; Ohsaki and Katoh [3]). However, in practical situation, the cross-sectional properties are selected from the discrete list of available sections. Therefore, the problem turns out to be a Mixed Integer Programming (MIP) problem (Stolpe and Svanberg [4]).

The topology optimization of continuum discretized to finite elements also has integer variables indicating existence and non-existence of the material. Although the problem is usually reformulated with continuous variable, the problem with stress constraint should be rigorously formulated in the form of MIP problem to account for the disappearance of stress constraint in the element with vanishing material.

In this study, a MIP approach is presented for topology optimization of trusses with discrete variables, and plates discretized to finite elements and subjected to in-plane loads, to minimize the total structural volume under constraints on stress and displacement.

\section{MIP formulation of topology optimization under stress constraints}

Consider first a pin-jointed truss that transmits axial forces only. Let $a_{i}$ and $l_{i}$ denote the cross-sectional area and length of the $i$ th member of a truss. $a_{i}$ is selected from the set of available sections as $a_{i} \in\left\{0, a_{i 1}, \ldots, a_{i N_{i}}\right\}$, $(i=1, \ldots, m)$, where $m$ is the number of members, and $N_{i}$ is the number of sections for the $i$ thember. A $0-1$ variable $x_{i k}$ is defined as

$$
x_{i k}= \begin{cases}1 & \text { if } a_{i}=a_{i k} \\ 0 & \text { otherwise }\end{cases}
$$


The nodal displacement vector against the static loads $\mathbf{f}$ is denoted by $\mathbf{u}$. The member elongation $c_{i}$ is written with respect to $\mathbf{u}$ as $c_{i}=\mathbf{b}_{i}^{\mathrm{T}} \mathbf{u}$, where $\mathbf{b}_{i}$ is a constant vector. The axial force vector is denoted by $\mathbf{s}=\left(s_{1}, \ldots, s_{m}\right)^{\mathrm{T}}$ with $s_{i}=\sum_{k=1}^{N_{i}} s_{i k}$. Let $\mathbf{u}^{\min }=\left(u_{1}^{\min }, \ldots, u_{n}^{\min }\right)^{\mathrm{T}}$ and $\mathbf{u}^{\max }=\left(u_{1}^{\max }, \ldots, u_{n}^{\max }\right)^{\mathrm{T}}$ denote the vectors of lower and upper bounds of displacements, where $n$ is the number of degrees of freedom. $c^{\min }$ and $c^{\max }$ are defined as appropriate lower and upper bounds of member elongation corresponding to the displacements satisfying $\mathbf{u}^{\min } \leq \mathbf{u} \leq \mathbf{u}^{\max }$. The equilibrium matrix between $\mathbf{s}$ and $\mathbf{f}$ is denoted by $\mathbf{B}$. The objective function is the total structural volume $V$. Then the topology optimization problem is formulated as

$$
\begin{array}{ll}
\text { Minimize } & V=\sum_{i=1}^{m} \sum_{k=1}^{N_{i}} x_{i k} a_{i k} l_{i} \\
\text { subject to } & \mathbf{B s}=\mathbf{f} \\
& x_{i k} a_{i k} \sigma_{i}^{\min } \leq s_{i k} \leq x_{i k} a_{i k} \sigma_{i}^{\max }, \quad\left(i=1, \ldots, m ; k=1, \ldots, N_{i}\right) \\
& u_{i}^{\min } \leq u_{i} \leq u_{i}^{\max }, \quad(i=1, \ldots, n) \\
& x_{i k} c^{\min } \leq s_{i k} \leq x_{i k} c^{\max },\left(i=1, \ldots, m ; k=1, \ldots, N_{i}\right) \\
& \left(1-x_{i k}\right) c^{\min } \leq a_{i k} \frac{E}{l_{i}} \mathbf{b}_{j}^{\mathrm{T}} \mathbf{u}-s_{i k} \leq\left(1-x_{i k}\right) c^{\max }, \quad\left(i=1, \ldots, m ; k=1, \ldots, N_{i}\right) \\
& \sum_{k=1}^{N_{i}} x_{i k}=x_{i} \leq 1,(i=1, \ldots, m) \\
& x_{i k} \in\{0,1\}, \quad\left(i=1, \ldots, m ; k=1, \ldots, N_{i}\right)
\end{array}
$$

where $\sigma_{i}^{\min }$ and $\sigma_{i}^{\max }$ are the lower and upper bounds of stress of the $i$ th member, and $E$ is the elastic modulus.

Although the details are omitted, the topology optimization problem of a plate (sheet) subjected to in-plane loads is formulated in MIP form in a similar manner as the truss topology optimization problem, where conservative linear bounds are used for von Mises stress. In the numerical examples, the MIP problem is solved by using the library CPLEX 10.2 [5].

\section{Numerical examples}

Consider a plane truss as shown in Figure 1, which has many members as an assemblage of the four traditional layouts, namely, Howe-truss, Platt-truss, Warren-truss and K-truss, as shown in Figure 2(a)-(d), respectively. The truss represents one of the two symmetric parts of a simply supported beam truss. Uniform loads $f$ are applied at the lower nodes. The members are appropriately divided into groups so that one of the four types is selected for each unit. The parameters are $h=2.0, w=24.0, E=2.0$ and $\sigma_{i}^{\max }=0.003$ for all members. The lower-bound stress is given by $\sigma_{i}^{\min }=-\left(l_{i}^{2} / l_{\min }^{2}\right) \sigma_{i}^{\max }$ to incorporate member buckling, where $l_{\min }$ is the length of the shortest member. The cross-sectional areas are chosen from the list $\{0.0,1.0,1.8\}$.

The optimal solutions are found for Cases 1-3 with three different load magnitudes $0.00007,0.0001$ and 0.00014 , respectively. The optimal topologies are as shown in Figures 2(a)-(c), where the width of each member is proportional to its cross-sectional area, and the CPU time (sec.) and the optimal objective value are shown in each figure. As is seen, Warren-truss tends to be selected for smaller load, while Howe-truss is selected for larger load. Note that CPU time strongly depends on the load magnitude; i.e. computational cost is smaller for larger load, because the number of admissible set of cross-sectional areas decreases as the load is increased.

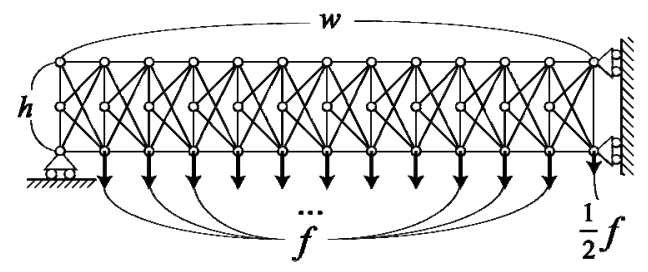

Figure 1: A plane truss subjected to static loads. 


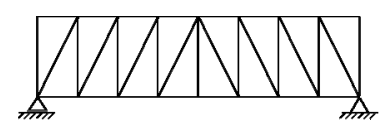

(a) Howe-truss

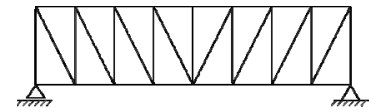

(b) Platt-truss

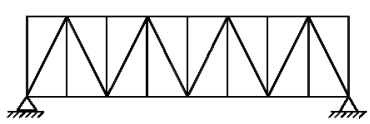

(c) Warren-truss

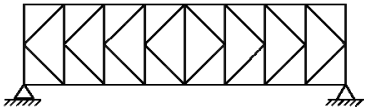

(d) K-truss

Figure 2: Four traditional types of plane truss.

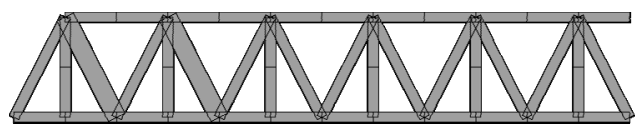

(a) Case 1: $\mathrm{CPU}=67900, V=65.410$.

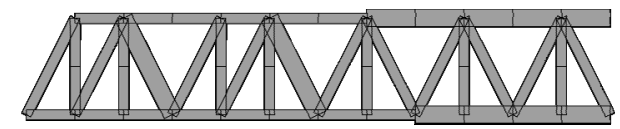

(b) Case 2: $\mathrm{CPU}=14600, V=74.610$.

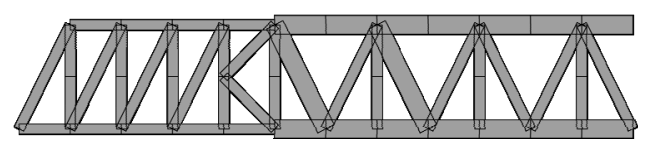

(c) Case 3: $\mathrm{CPU}=10600, V=81.202$.

Figure 3: Optimal topologies and cross-sectional areas of the plane truss.

We next optimize a dome truss as an assemblage of the Schwedler-dome and Lamella-dome as shown in Figure 4(a) and (b), respectively. The cross-sectional areas are selected from the list $\{0.0,1.0,2.0\}$, and we consider Cases 1-4 with load values 0.00001, 0.00003, 0.00005 and 0.0001, respectively. The optimal topologies are as shown in Figures 5(a)-(d). As is seen, the optimal truss is a Lamella-dome for Case 1 with smallest load, while Schuwedler-dome dominates for larger load magnitudes. CPU times (sec.) for Cases 1-4 are 3.5, 47.9, 511 and 669 , respectively.

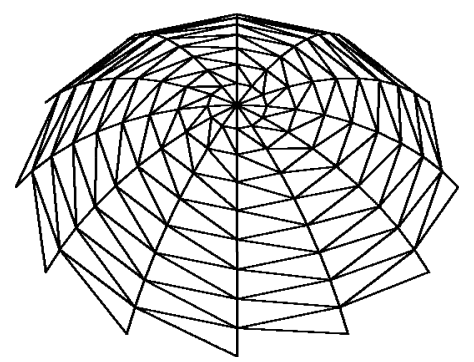

(a) Schuwedler-dome

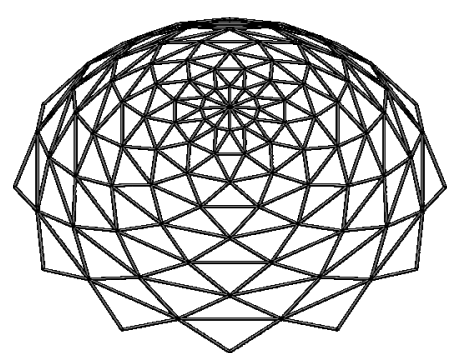

(b) Lamella-dome

Figure 4: Two traditional types of space truss.

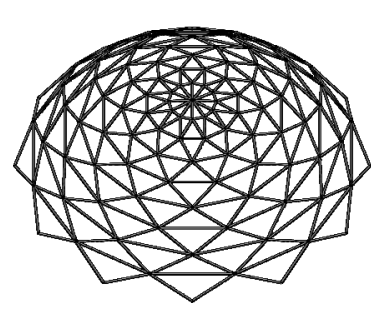

(a) Case 1: $V=1404.3$.

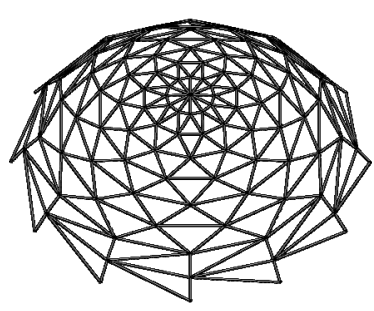

(b) Case 2: $V=1441.1$.

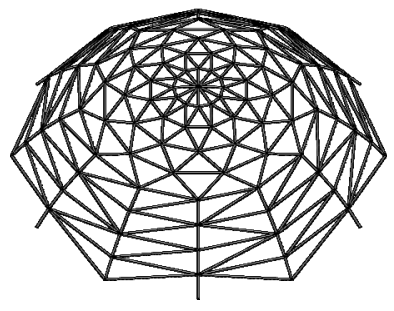

(c) Case 3: $V=1474.4$.

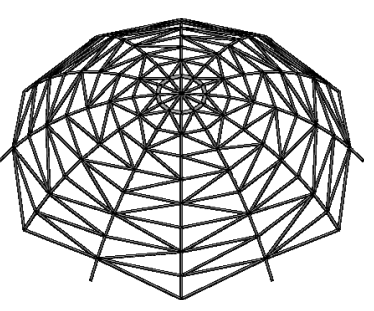

(d) Case 4: $V=1527.2$.

Figure 5: Optimal topologies and cross-sectional areas of the space dome truss. 
Consider next the plate as shown in Figure 5 subjected to in-plane loads, where $f=3.0$, the size of the grid is 1.0 , the elastic modulus is 2.0, Poisson's ratio is 0.25 , and the upper-bound stress is 3.0 The objective function of the total structural volume, and compliance multiplied by small penalty coefficient $\rho=1.0 \times 10^{-4}$ is added to prevent non-uniqueness of the solution that drastically deteriorates the convergence property of MIP.

Optimization is carried out iteratively through small regions $D_{1}, \ldots, D_{9}$ indicated in Figure 6. Approximate optimal topologies are found for Cases 1-3, where different sequences of the optimizing regions are used for Cases 1 and 2, and compliance is not added in Case 3, and a checkerboard filter is used for all cases. Optimal topologies are shown in Figure 7(a)-(c). Note that Case 1 and 2 have the same objective value. The CPU times (sec.) are 2990 and 4060 for Cases 1 and 2, respectively, while a solution with $V=35$ was found in 360000 sec., if the whole region is to be optimized simultaneously.

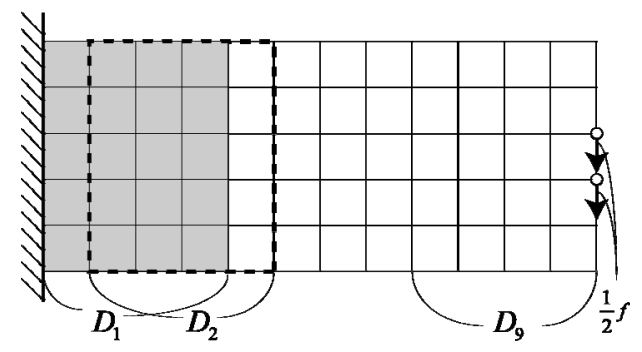

Figure 6: A plate model subjected to in-plane loads.

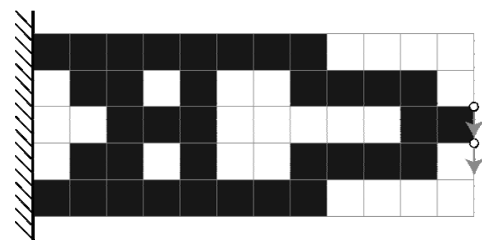

(a) Case 1: $V=35$.

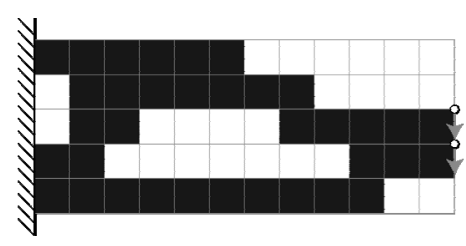

(b) Case 2: $V=35$.

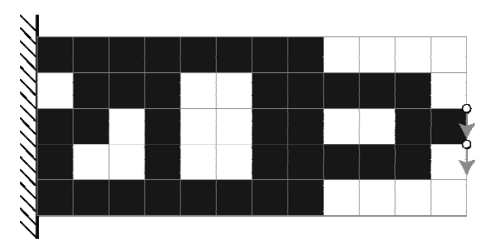

(c) Case 3: $V=36$.

Figure 7: Approximate optimal topology of plate.

\section{Conclusions}

Optimal topologies have been found for trusses and plates by MIP formulation. It has been shown that new topologies of plane trusses and space dome trusses as assemblage of traditional layouts can be successfully found by solving the MIP problem. Approximate optimal topologies of plates under stress constraints can be found by iteratively optimizing the substructures.

\section{References}

[1] Bendsøe MP and Sigmund O. Topology Optimization: Theory, Methods and Applications, Springer, 2003.

[2] Hagishita T and Ohsaki M. Topology optimization of trusses by growing ground structure approach, Struct. Multidisc., in press.

[3] Ohsaki M and Katoh N., Topology optimization of trusses with stress and local constraints on nodal stability and member intersection, Struct. Multidisc. Optim., Vol. 29, pp. 190-197, 2005.

[4] Stolpe M and Svanberg K. Modeling topology optimization problems as linear mixed 0-1 programs, Int. J. Numer. Meth. Eng., Vol. 57, pp. 723-739, 2003.

[5] ILOG, ILOG CPLEX 10.2 User's Manual, 2007. 


\title{
Optimal design of glass grid shells with quadrilateral elements by means of a genetic algorithm.
}

\author{
Mario SASSONE*, Alberto PUGNALE \\ *Assistant Professor, Department of Structural and Geotechnical Engineering, Politecnico di Torino \\ 10125 - Viale Mattioli 39, Torino, Italy \\ mario.sassone@polito.it
}

\begin{abstract}
In this paper a constructional problem related to grid-shells design is approached as an optimisation problem and an evolutionary solution technique is proposed. The construction of glass grid-shells, when only four sides cladding elements are used, requires to check the planarity of each element, it means that the four corner points have to belong to the same plane. This requirement can be satisfied by generating the grid surface in specific ways (Schlaich and Bergermann [5]), but when the design involves truly free form shapes, a procedure of optimisation able to reduce the non planarity of each element can be applied. The proposed one is based on the use of a genetic algorithm and has been applied to a benchmark and to a real case, in order to evaluate the efficiency of the procedure and the goodness of the solution.
\end{abstract}

\section{Introduction}

The design of complexes structures involves not only complexes analyses and verifications but even the solution of constructional problems. Especially in free form architecture, when members and elements shapes are not regular, many manufacturing problems have to be solved, in order to make the construction efficient and to reduce costs. In recent years, due to the large diffusion of numeric control machine tools, the production problems transformed into design problems and a the process of manufacturing needs to be managed almost completely at the design time. The effect on architecture is the growth of free form projects, made possible by a strong industrialisation and prefabrication of the components, but non necessarily in the sense of a request of standardisation. Hence the design of free form architecture usually involves the search of optimal solutions of design problems and the development and use of robust and flexible computational tools, as the one presented in this paper, can give a significant contribution.

\section{The planarity problem in grid shell design}

The geometry of a grid shell is based on meshing the reference surface with plane polygonal faces. The use of triangles is the more general solution, allowing to mesh each kind of free form surface. From the construction point of view it can be expensive, due to the complexity of structural joints, that require a six way link at each node, and to the cuts in glass sheets that produce a large amount of scrap. A more efficient solution is the use of quadrilaterals, as developed and applied by Schlaich, provided that a suitable bracing system is adopted. The use of pentagons and hexagons is almost limited to structures based on the mesh of the Fuller's dome, as the well known Eden Project designed by Grimshaw and Partners. When polygons with more than three sides are used, it is necessary to evaluate the planarity of the vertexes, it means that all the corner points must lay on the same plane. The cladding glass elements, indeed, are cut from plane sheets so that the joints between structure and glass must respect the planarity. The curved or double curved glasses, obtained by hot forming, are still to much expensive to be adopted in current grid shell design. The planarity requirement, on the other hand, lose its importance if the cladding material is soft and can be shaped freely, as in the case of inflated ETFE pillows. The planarity of grid shells made of quadrilaterals can be obtained by adopting specific shape generation rules (Schlaich and Bergermann [5]) based on translation and scaling of curves. If a spatial curve is translated in 
space following another spatial curve, it is easy to show that the surface one obtains has the property, when meshed by curves parallel to the generating ones, of being divided only in plane quadrilaterals; this property is still valid if the translating curve is uniformly scaled during translation. Following this approach the problem is solved by shrinking the field of possible shapes, so that it becomes hard to deal with truly free form architectures. The procedure presented in this paper, on the other hand, is based on an optimization process, by means of an evolutionary genetic algorithm, that can be applied to the solution of a wide set of problems, independently of the global shape or the polygon number of sides. The convergence of the solver to the optimal solution is guaranteed by the robustness of the algorithm; when the problem is underdeterminate and more than one optimal solution are possible, the procedure can be used to explore empirically the range of optimal solutions.

\section{Genetic representation of the problem}

When a genetic technique is used in dealing with optimization, the main problem is the choice of a correct and appropriate description of the solution. Many studies have been made about encoding methods and their behaviour (Antonisse [1], Janikow and Michalewicz [3], Wright [9]) with different results. It is generally agreed, anyway, that the efficiency largely depends on the problem characteristics and on the details of the adopted GA (Mitchell [4]). Besides the binary coding, based on the construction of a kind of binary chromosomes, even integer and real description of variables are possible. When the optimization problem concerns the search of an spatial configuration, described by a set of coordinates or control points or other geometric parameters, the representation of the solution inside the algorithm should maintain the topological structure of the problem. If, for instance, the problem deals with the search of a spatial surface having specified properties, the solution can be represented inside the algorithm as a two dimensional matrix of interpolation points or of NURBS control points, so that the reproduction operators can take into account the topological properties of the problem. The performance of a solution depends on the absolute geometrical position of each point, as well as on the relative position between on point and the ones close to it. Preserving such neighbourhood relations, by maintaining the correspondence between the geometrical morphology and the representation scheme of the individuals (solutions) is a good way to improve the algorithm operators efficiency.
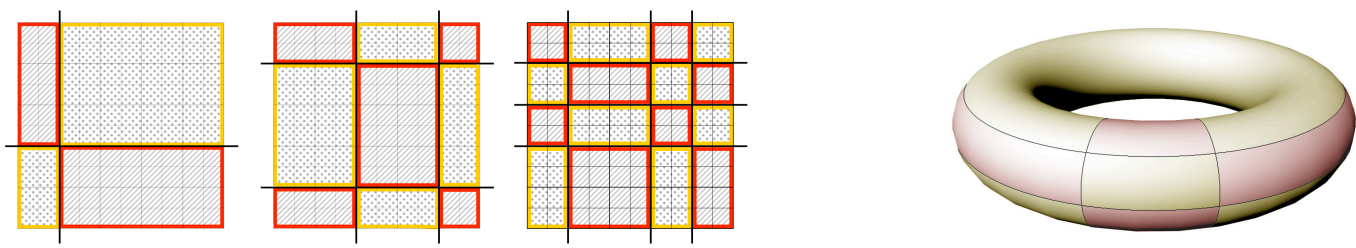

Figure 1: Topological crossovers

In the present case the shape of the grid shell is represented by a matrix of coordinates, i.e. a two dimensional matrix in which each element stores the $\mathrm{x}, \mathrm{y}$, and $\mathrm{z}$ coordinates of the grid nodes, as real numbers. The use of a real numbers in coding the design variables is part of the adopted evolutionary strategy. In fact, the way information are coded interacts with the reproduction operators, as the crossover operator, so that it can work in different ways. If a spatial coordinate (or vector of coordinates) is binary coded, the action of the crossover operator can "cut" the code and generate new values for the spatial coordinate. If the coordinate is coded as a real number, the crossover can not create new values of the coordinate, but only exchange the coordinates values from one point of the grid to the other. Coding the point coordinates as real numbers, is then a way to separate the generation of new values (that is performed by the random mutation operators) from the construction of new solutions by combining the previous ones (reproduction). A correct tuning of the operators is then necessary to guarantee the balance between the continuous exploration of the solutions domain and the convergence towards the optimal. A rapid convergence of the algorithm could fall into a local minimum, known as a genetic drift. On the other hand, an high exploration of the domain could drastically reduce the algorithm efficiency, contrasting the convergence wasting some good sub-optimal solutions. As a rule to select the individuals in the population that will create offspring for the next generation, and the number of new individuals, the classical fitness-proportionate selection with "Roulette Wheel" method is used. In order to 
guarantee a continuous increase of the best individual performance the Elitism selection method is implemented too: at each generation the individuals with the best fitness value are kept intact for the next one, so that the best fitness value can not decrease as an effect of the crossover or of mutations.

\section{The performance evaluation}

In order to quantify the non-planarity of a set of four points, the distance of the fourth point from the plane defined by the first three is calculated for each quadrilateral element of the configuration. The vector containing such values represents the difference between the actual solution and one of the optimal ones. The reciprocal of the vector norm is then a measure of the fitness and the goal of the optimisation is to minimise that value. The use of different kind of norms can slightly affect the convergence process: the Euclidean norm is more influenced by the global shape of the pattern, meanwhile higher degree p-norm is more sensible to local peaks of non planarity, i.e. to local deviations from the desired shape.

\section{Applications and results}

A group of applications has been developed as a reference for tuning the algorithm. In this cases the number of design variables describing the geometric configurations is relatively small and at least one solution of the problem is known to be existing. That allows the comparison between the theoretical solution and the one obtained by the algorithm, under different conditions. One of these benchmark applications is depicted in figure 2. The solution domain is defined starting from a surface with constant cross section, that satisfies the planarity condition when meshed following the directrices and the generatrices curves. Hence the problem is to find one of this solution starting from a randomly generated set of points. In figure 2 - left is depicted one of the individuals of the first random generation, meanwhile in figure 2 - centre the best performing pattern after the optimization process is depicted. On the right the best fitness at each generation (black line) and the average fitness at each generation (red line) are shown. In this optimisation process only the vertical coordinate of each point is assumed as a design variable, so that the plan projection of the points is constant. Different results can be obtained if all the three coordinates can change, or even if the points are forced to move on an assigned surface. In this case the optimal pattern, when it exists, is generally smooth as the underlying surface, but a scattering appears in the horizontal position, making the grid shell look very irregular.
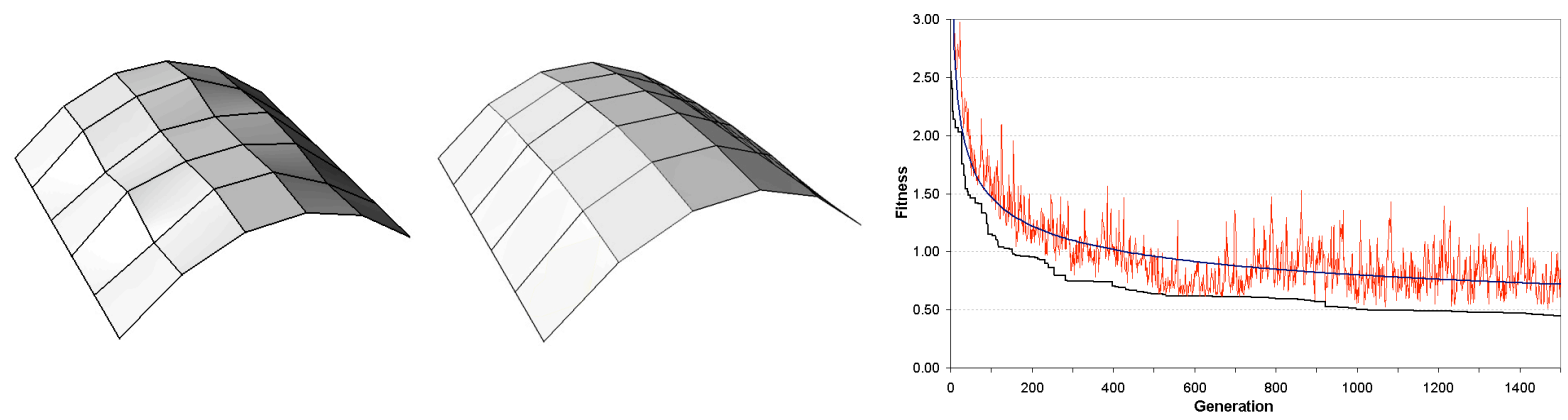

Figure 2: Barrel vault: starting shape; shape after 1500 iterations; average and minimal fitness

After the algorithm has been applied to the benchmark application and the best values have been assigned to the parameters, the optimization procedure has been applied to a real case. In figure 3 - left the a 3D design view of a grid shell is shown. The space to be covered is a cross shaped gallery inside an historical building. The roof has two symmetry axes, so the optimization process has been applied only to one quarter of the whole structure. The global shape of the glass roof has been defined by the architects in order to emphasise the central space and to reach the maximum transparency effect. Hence the domain of the design variables is relatively narrow, limiting the search to the solutions that are close to the desired shape. Following a distinction that has already been introduced in Sassone and Pugnale [8], it can be considered a process of form - improvement, rather than a process of form - finding. Different sets of design variables have been adopted, representing different ways to explore the solutions space: in figure 3 - left one of the starting pattern of the process is compared with the solution obtained after 4500 iterations. The problem in this case is largely underdeterminate, due to the fact that assuming all the three point coordinate as variables a huge number of optimal solution can be obtained. The one 
shown in figure looks particularly irregular, with trapezoidal and skewed elements, but, of course, each element is perfectly plane.
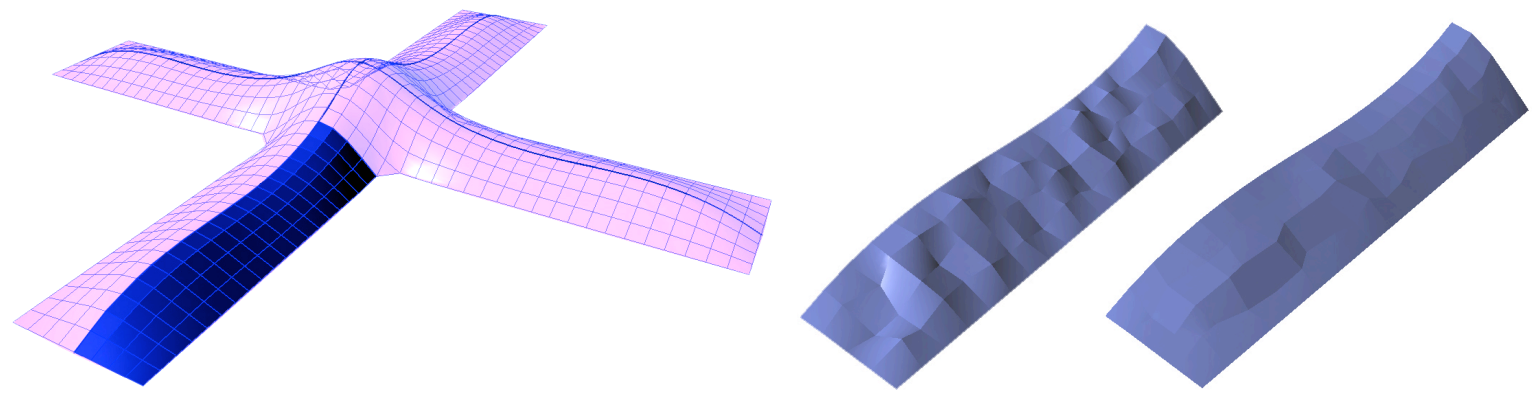

Figure 3: Urban gallery glass roof: sketch of the gallery roof; random shape; shape after 4500 iterations

\section{Future developments of the research}

The main feature of a genetic or generally speaking evolutionary optimisation algorithm is its very strong capacity to converge to optimal or sub-optimal solutions. When the problem is particularly hard the process can take long time before reaching solutions interesting from the applicative point of view. The research will hence continue in working on the efficiency of algorithms and by applying to architectural and structural problems even other evolutionary techniques, as for instance the particle swarm method or the leap-frog method, that have already yield to significant results in different fields of application. Secondly, this approach allows interaction between different problems, in the multi-objective optimisation, so that other kind of performance, as the structural behaviour (Pugnale and Sassone [5]) can be optimised together with the constructional one.

\section{References}

[1] Antonisse J. A new interpretation of schema notation that overturns the binary encoding constraint. In Proceedings of the Third International Conference on Genetic Algorithms, Schaffer JD (eds.) Morgan Kaufmann: San Francisco, 1989; 86-91.

[2] Holgate A. The Art of Structural Engineering: The Work of Jörg Schlaich and his Team. Edition Axel Menges, 1997.

[3] Janikow CZ, Michalewicz Z. An experimental comparison of binary and floating point representations in genetic algorithms. In Proceedings of the Fourth International Conference on Genetic Algorithms, Belew RK, Booker LB (eds.) Morgan Kaufmann: San Francisco, 1991; 31-36.

[4] Mitchell M. An introduction to Genetic Algorithms. The MIT Press, 1998.

[5] Pugnale A, Sassone M. Morphogenesis And Structural Optimization of Shell Structures with the aid of a Genetic Algorithm. Journal of the International Association for Shell and Spatial Structures 2007; 48: 161-166.

[6] Renner G, Ekàrt A. Genetic Algorithms in Computer Aided Design. Computer Aided Design 2003, 35: 709-726.

[7] Sassone M. Geometry and Structure Generation in Grid Shell Design. In International Workshop on Computational Morphogenesis, Sub-Working Group TG13 of IASS: Nagoya (Japan), 2006; 11-12.

[8] Sassone M, Pugnale A. Structural optimization of shell spatial structures with a genetic algorithm. In Special Workshop: Advanced Numerical Analysis of Shell-Like Structures, Croatian Society of Mechanics (HRV): Zagreb (Croatia), 2007; 247-257.

[9] Wright A. Genetic algorithms for real parameter optimization. In Foundations of Genetic Algorithms, Rawlins G (eds.) Morgan Kaufmann: San Francisco, 1991. 
Proceedings of the 6th International Conference on Computation of Shell and Spatial Structures

IASS-IACM 2008: "Spanning Nano to Mega"

28-31 May 2008, Cornell University, Ithaca, NY, USA

John F. ABEL and J. Robert COOKE (eds.)

\title{
Development of intelligent truss optimization system
}

\author{
Seung-Chang LEE*, Jung-Keun $\mathrm{OH}$ \\ *Senior Researcher, Ph.D. \\ Research Institute of Technology, Samsung Corporation (Eng. \& Const. Div.), Seoul, Korea \\ sc88.1ee@samsung.com
}

\begin{abstract}
The purpose of this paper is to implement the Intelligent Truss Optimization System for Samsung named ITOSS. The system has the function from generating the configuration to analyzing the nonlinear behavior. For this, the system is composed of four modules; that is, configuration module, property and loading module, optimization module and nonlinear analysis module. In the configuration module, it can automatically generate the geometry of flat roof, vault roof, circular dome and elliptical dome with the basic data. Property and loading module has the automatic function that wind or other loads can be subjected to the surface of truss or dome. In the optimization module, the expected global minimum can be arrived without stop through the re-analysis and reselection automatically. In the nonlinear analysis module, it can check the snap-through behavior for the dome structure with low rise.
\end{abstract}

\section{Introduction}

There are some differences of structural considerations between large spatial structure and normal building structure. First, the structural behavior of roof or dome is determined by either snow or wind load. Second, various boundary conditions such as sliding should be provided for the overall stability of long span structure. Third, the connection between members is importantly checked because it requires the proper connection detail to resist the local buckling. Fourth, the roof truss has experienced various boundary conditions and inverse member forces with respect to the final design status during assembling and erecting.

Because of these differences and complexities in the structural design of dome structure, the outcome by novice engineer can occasionally include some mistakes that lead to the design and construction failures. In order to prevent those failures, both novice and professional engineers need much time in order to check truss structure composed of many members. Many engineers require the automated optimization system that can consistently reach at the optimum solution with high quality within the prescribed time.

In this study, the Intelligent Truss Optimization System for Samsung named ITOSS; the system is developed for practice. The system has the function from generating the configuration to analyzing the nonlinear behavior. For this, the system is composed of four modules; that is, configuration module, property and loading module, optimization module and nonlinear analysis module. In the configuration module, it can automatically generate the geometry of flat roof, vault roof, circular dome and elliptical dome with the basic data. Property and loading module has the automatic function that wind or other loads can be subjected to the surface of truss and dome. In the optimization module, the expected global minimum can be arrived without stop through the re-analysis and re-selection automatically. In the nonlinear analysis module, it can check the snap-through behavior for the dome structure with low rise.

\section{Configuration module}

In the preliminary design of dome structure, designer and structural engineer want to try to make various configurations as alternatives. It is not hard to produce the configuration of dome by CAD, but it is timeconsuming. Thus, the need of automated program is increased to generate the configuration of dome within real 
time. Using this module and ITOSS, structural engineer can ultimately get the optimum configuration which can satisfy the structural guidelines and architect's requirements.

In this study, the configuration of dome is generated from the equation of an ellipsoid as in Equation (1) in an $x-$ y-z Cartesian coordinate system.

$$
\frac{x^{2}}{a^{2}}+\frac{y^{2}}{b^{2}}+\frac{z^{2}}{c^{2}}=1
$$

where $\mathrm{a}$ and $\mathrm{b}$ are the equatorial radii (along the $\mathrm{x}$ and $\mathrm{y}$ axes) and $\mathrm{c}$ is the polar radius (along the $\mathrm{z}$ axis). If all three radii are equal, the solid body is a sphere. If two radii are equal, the ellipsoid is a spheroid. The points $( \pm \mathrm{a}$, $0,0),(0, \pm b, 0),(0,0, \pm c)$ lie on the surface. The line segments are created from the origin to these points. Thus, the configuration of circular dome is generated from the sphere. The configuration of oblate and elliptical dome is based on that of oblate and elliptical spheroid. In addition, the configuration module of ITOSS produces flattype and vault-type roof. Figure 1 through Figure 4 show the input menu to generate the configuration of flat roof, vault roof, circular dome and elliptical dome. The input menu is an easy-to-use due to the graphical description. It requires only basic and the least information, for example, diameter, height and number of ring is needed to generate the dome.

\section{Property and loading module}

Most finite element programs can easily assign element type, the sectional and material data of members, because they have graphic-based preprocessor. Easier assigning is possible in the ITOSS. The element type of all members can be selected of either beam elements or bar elements by one click. The sectional and material property of initial member can be set automatically from sectional database and input menu. Initial member with the selected sectional and material property will be used for preliminary analysis.

The design of dome structure is started with two kinds of boundary conditions; sliding or hinge condition. ITOSS can automatically assign those boundary conditions to the perimeter of dome as initially assumed boundary condition (Figure 5).

There are two principal loads that govern the structural design of dome. One is snow load subjected to the dome with low rise to span ratio. The other is wind load subjected to the dome structure with high rise-to-span ratio. Regulations for wind load are complicated in the dome structure. It is not easy to assign proper wind load to the dome's surface. In the ITOSS, the input of wind's load value and its assigning to the surface of the structure are implemented as shown in Figure 6.

Based on ASD or LRFD code, the load combinations can be generated from the input menu. These combinations will be provided in the optimization design.

\section{Optimization module}

The optimization module is based on the trial and error method which has been widely used in the practice. Trial and error method is more efficient than other optimization methods when it considers practical conditions; that is, discrete design variables from member database and member grouping technique.

Figure 7 shows the trial and error method applied in the ITOSS. The procedure starts with randomly selected member size. Initial member size is set with the 0.8 of allowable stress ratio from the preliminary analysis results. Starting points composed of initially set members satisfies the stress constraint but do not consider the displacement constraint. After re-analyzing with initial set members, all members are checked with both stress and displacement constraints. If the constraint violation is occurred at one or more members, the size of those members will be increased to upper size until the satisfaction. When all constraints are satisfied, member size set is generated and saved as a possible optimum result. After saving possible optimum result, all member sizes are decreased to lower sizes. Amount of reduced size is problem-dependent and randomly selected, for example $10^{\text {th }}$ lower rank than current size in member database. The function of reduction is to provide another starting point to reach better solution. However the reduced size will be increased to upper size until all constraints are satisfied through re-analysis, stress and displacement check procedure. Therefore the procedure could give another possible optimum solution. In the last stage, we could get the best solution from the possible solution by some trials. This is a kind of multi-start procedures to arrive at global minimum. 


\section{Nonlinear analysis module}

As the load is increased, shallow dome structure might be in the situation where the elastic stiffness of the structure is reduced by the effects of compressive stress. If this effect causes the structure to suddenly displace a large amount in a direction, normal to the load direction then it is classical bifurcation buckling. If there is a sudden large movement in the direction of the loading, it is snap-through buckling.

Nonlinear analysis module of ITOSS has been developed to consider geometric nonlinearity and material nonlinearity of dome structure. It can automatically convert linear model to nonlinear model. As shown in Figure 8, the eigen buckling analysis is performed before the nonlinear analysis. This can provide the linear buckling load of the structure which can be used for determining the options for nonlinear analysis. Figure 9 shows the menu for nonlinear analysis which can easily select the type of nonlinear and its options.

\section{Implementation and verification}

ITOSS has been implemented with LUSAS ${ }^{\mathrm{TM}}$ (LUSAS [1]) and its LPI function. To verify the ITOSS's performance, it is applied to the actual dome project with approximately 5000 members (Figure 10). The diameter of dome is about $35 \mathrm{~m}$ and its design is governed by wind load. In this paper, the optimization module of ITOSS is tested. ITOSS gives the satisfactory results of approximately $10 \%$ steel weight minimization compared to the manual optimization process.

\section{Conclusions}

The optimum design of dome structure is still a difficult task because it has a lot of members, various load cases and etc. It is extremely time-consuming process and the results by novice are very often not credible. Due to the development of ITOSS, it can generate credible optimization results within the prescribed time.

\section{Acknowledgement}

The authors wish to express their thanks to Mr. Jung and Han of FEA Korea for their great help in the programming of ITOSS.

\section{References}

[1] LUSAS. Theory and user manuals. Version 14.0. Surrey, UK: FEA Ltd, 2007

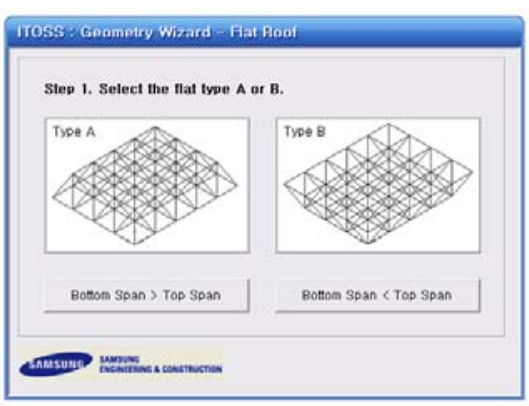

Figure 1: Configuration menu for flat roof

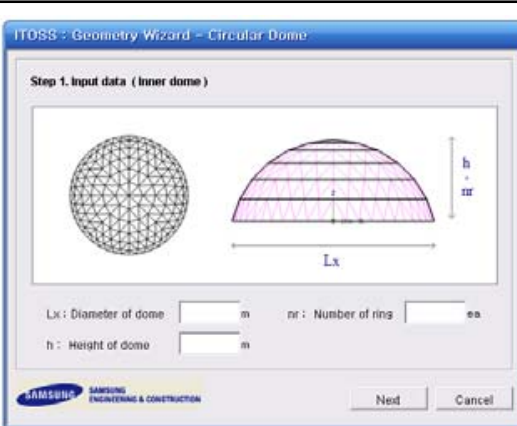

Figure 3: Configuration menu for circular dome

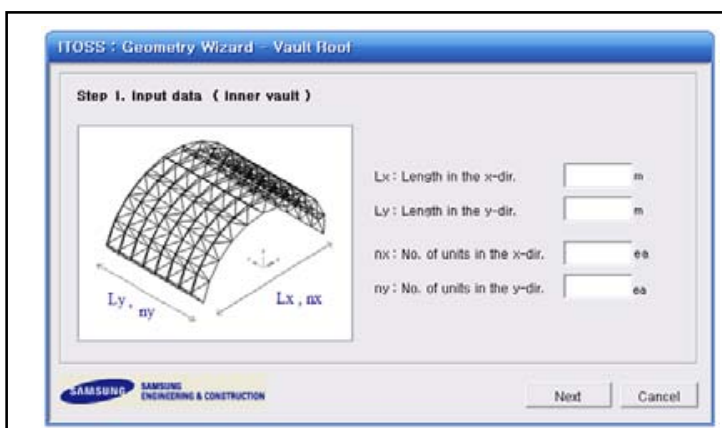

Figure 2: Configuration menu for vault roof

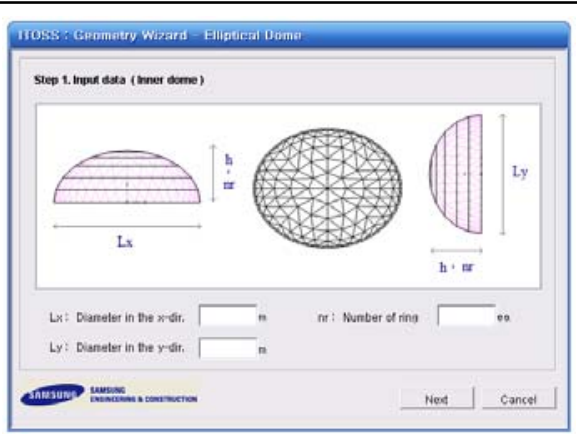

Figure 4: Configuration menu for Elliptical dome 


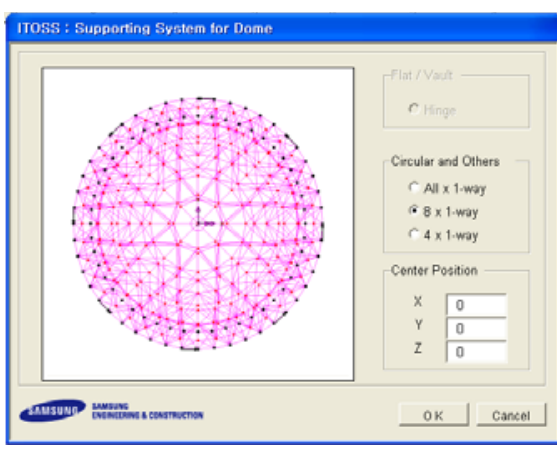

Figure 5: Automatic assigning boundary condition

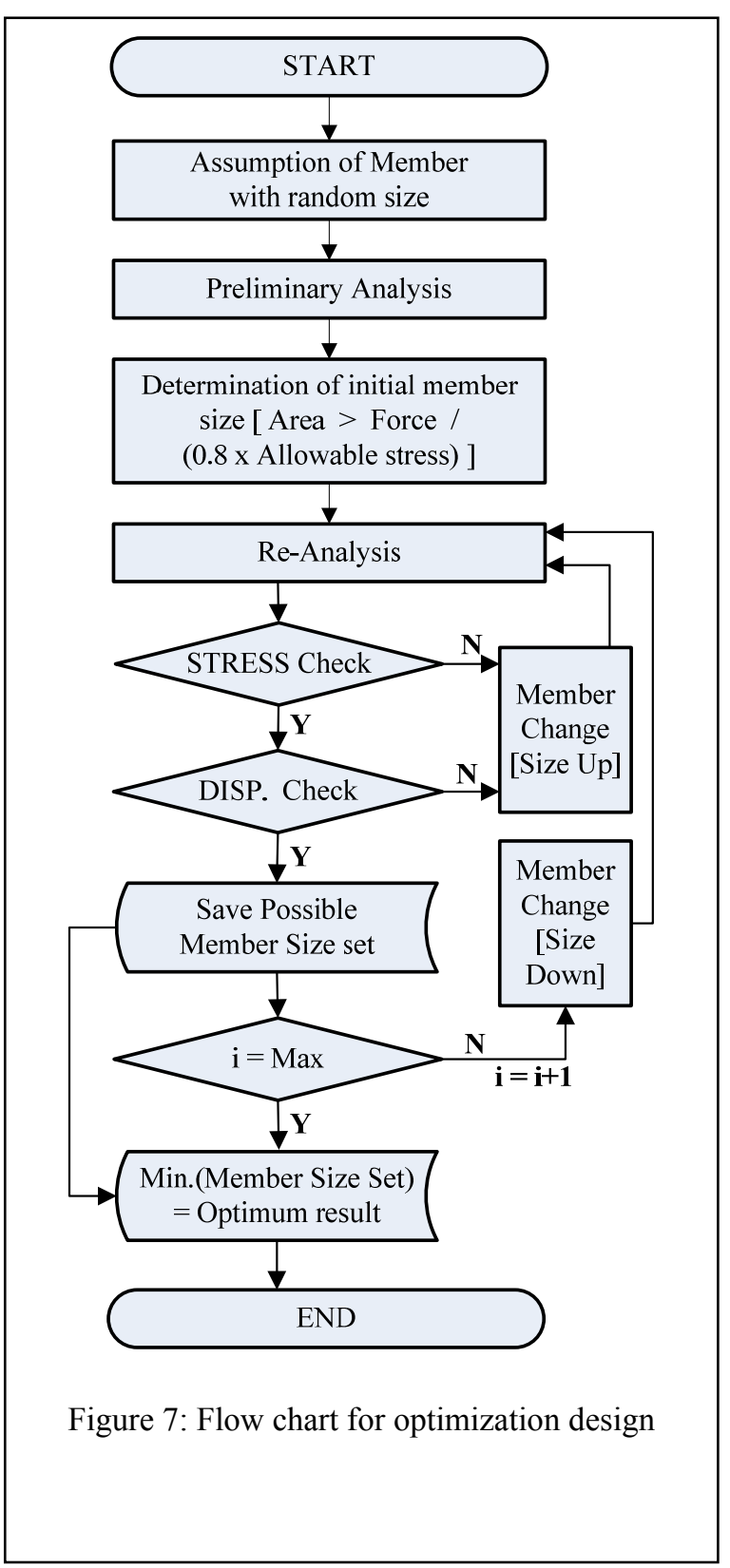

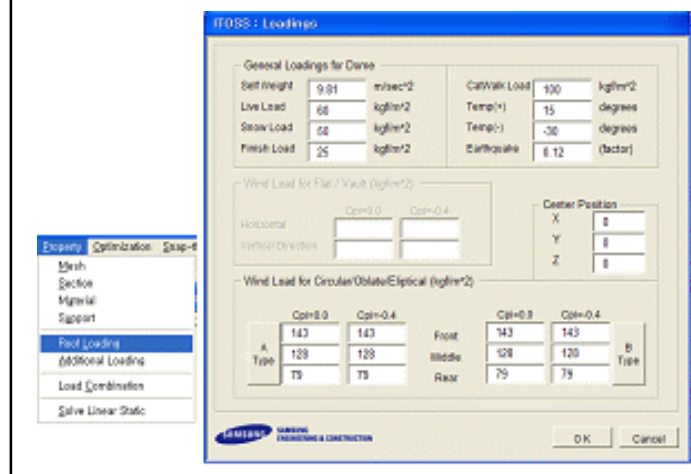

Figure 6: Input menu for wind load

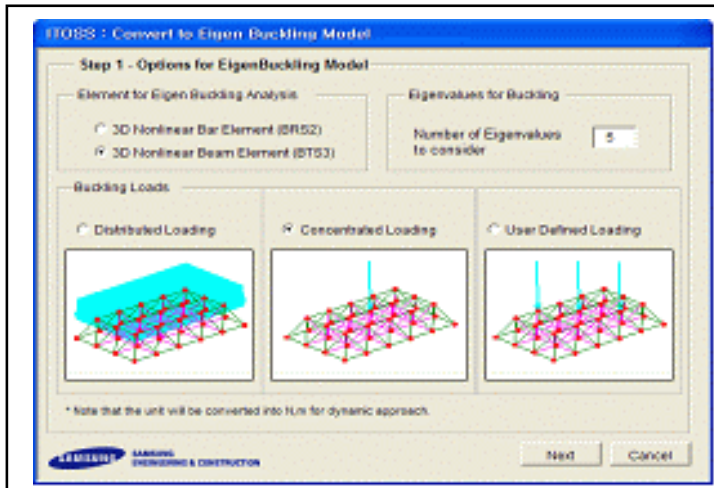

Figure 8: Input menu for eigenbuckling model

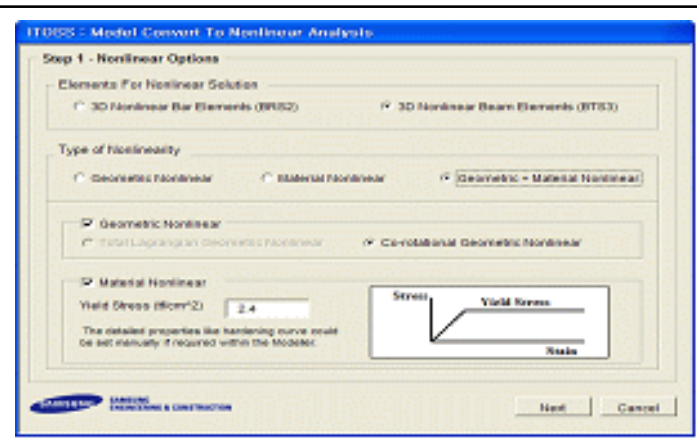

Figure 9: Input menu for nonlinear options

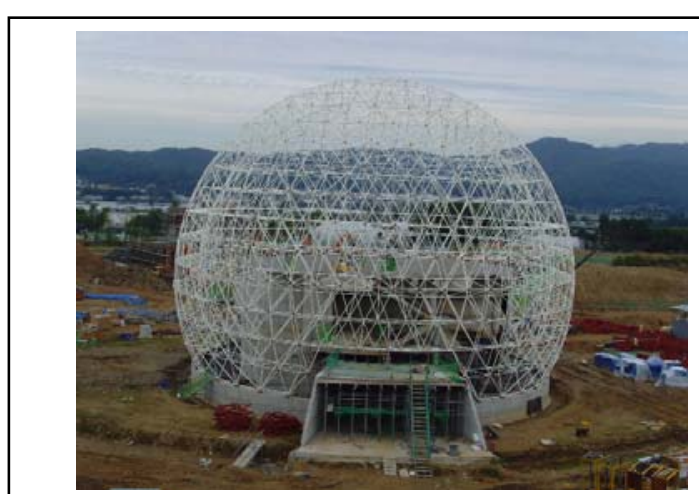

Figure 10: Actual dome project for verification 
Proceedings of the 6th International Conference on Computation of Shell and Spatial Structures

IASS-IACM 2008: "Spanning Nano to Mega"

28-31 May 2008, Cornell University, Ithaca, NY, USA

John F. ABEL and J. Robert COOKE (eds.)

\title{
Shell surface with curved fold lines inspired by paper folding art
}

\author{
$\underline{\text { Rohamezan ROHIM}}^{*}$, Kok Keong CHOONG, J.Y KIM \\ *Rohamezan Rohim \\ Faculty of Civil Engineering, \\ Universiti Teknologi MARA, Penang, Malaysia \\ mei_zan@yahoo.co.uk
}

\begin{abstract}
Paper folding art constitutes a rich source of idea for possible application to shell and spatial structures. The combination of flat surface with straight fold lines as seen in folded plate structures provides an excellent choice for possible adaptation as load carrying structures. Other than structural merit due to the folds, such folded plate surface has also the advantage of being aesthetic due to interplay of shadow on the surface caused by the existence of folds. In this paper, a novel type of folded surface called SSCFL where the fold lines are curved instead of straight are introduced. Procedures for producing folded object and computer generation of SSCFL models are proposed.
\end{abstract}

\section{Introduction}

The load carrying capacity of a flat and thin surface structure is rather limited. However, if a surface is folded, enhancement in stiffness and strength of the resulting folded surface can be achieved due to the increase in effective depth. Folded plate structure was first built as German coal bunker in early 1920s. Since then many types of folded plate have been studied, analysed and built (Ketchum, [6]). Besides roof structures, folded plates are also found in concrete stairs, folded lampshades and lanterns, corrugated sheet pile and others. In Japanese culture, the richness of the fold geometries can be seen in their art of paper folding or origami. Origami has been explored for application to scientific and mathematical problems such as packaging, deployment of satellite in space, deployed and distributed airbag and others [5].

In comparison with surface with straight fold lines, shell surface with curved fold lines has been less studied. Since the later surface combined the advantages of a shell surface and folds, it might be a very attractive alternative structural form to be considered. A.Razzack [1] has conducted a pioneer study on folded shell surface found in nature. The combination of surface with curve fold lines studied in this paper has been inspired by the work of Huffman $[3,4]$. Huffman [3] has outlined the fundamental of how papers or zero-curvature surfaces behave near the crease of a cone surface. A few samples of geometric paper folding, which can enhance the aesthetic of any folded surfaces due to the interplay of shadow have also been shown in the paper. Such form due to Huffman has been termed as shell surface with curved fold lines (SSCFL) in this paper. Inspired by the work of Huffman, Harun [2] has carried out measurement of model of SSCFL with the objective of obtaining basic data for further structural analysis. MD Resadi [7] has created a small-scale physical model of SSCFL in order to study the aspect of constructability.

Before SSCFL can be considered for structural application, its structural behaviour must be first understood. This study has been carried out with the objective of proposing a systematic procedures for the generation of SSCFL. The subsequently part of structural analysis is not treated.

\section{Shell surface with curved fold lines (SSCFL)}

\subsection{Folded surface by Huffman}

Huffman [4] has been able to produce complex three dimensional unit chains of cube and rhomboids, tesselations of triangular, pentagonal and star shaped blocks. Huffman developed a structure based around 


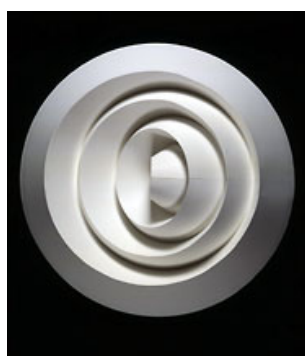

(a)

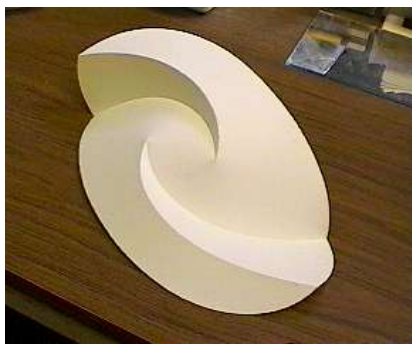

(b)

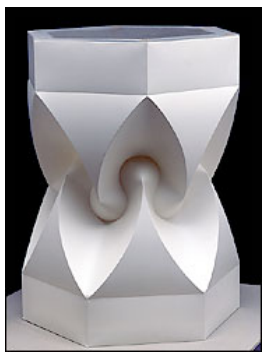

(c)

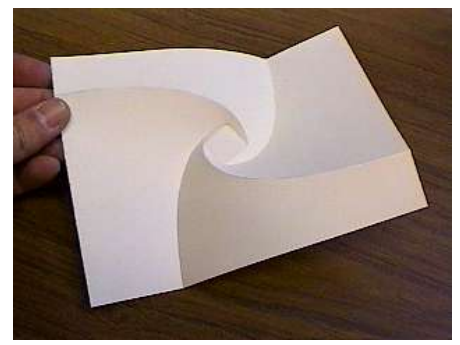

(d)

Figure 1: Curved lines with folds: a) An origami form of concentric domes, b) 4 parabolic curved fold through center, c) A tower like paper structure, d) 4 parabolic curved folds meet at center square

curved folds. Parabola and ellipse curves became the domain of his research (Wartheim, [8]). One of the main feature of Huffman's work is existence of curved fold lines on surface in contrast to traditional folded paper model with straight folds. Some of Huffman's ideas are shown in Figure 1. In this paper, the idea shown in Figure 1(d) has been selected as the study object.

\subsection{Folding process of SSCFL}

In order to produce a desired paper model of SSCFL, the following steps have been adopted (Harun, [2]). A piece of paper with a dimension of $\mathrm{BxB}$ is prepared. A center square of bxb is then drawn around on the paper as shown in Figure 2. Equation for the curved fold lines is next selected. In this study, curved fold lines in the form of parabolic curve, $y=a x^{2}+b x+c$, has been chosen. Three points are identified in order to determine the coefficient of the equation $a, b$ and $c$. This parabolic curve starts from point A1 and extends towards the paper edge. Other than point $A 1$, the parabolic curve passes through point $P_{1}$ and point $P_{2}$ as shown in Figure 2. After
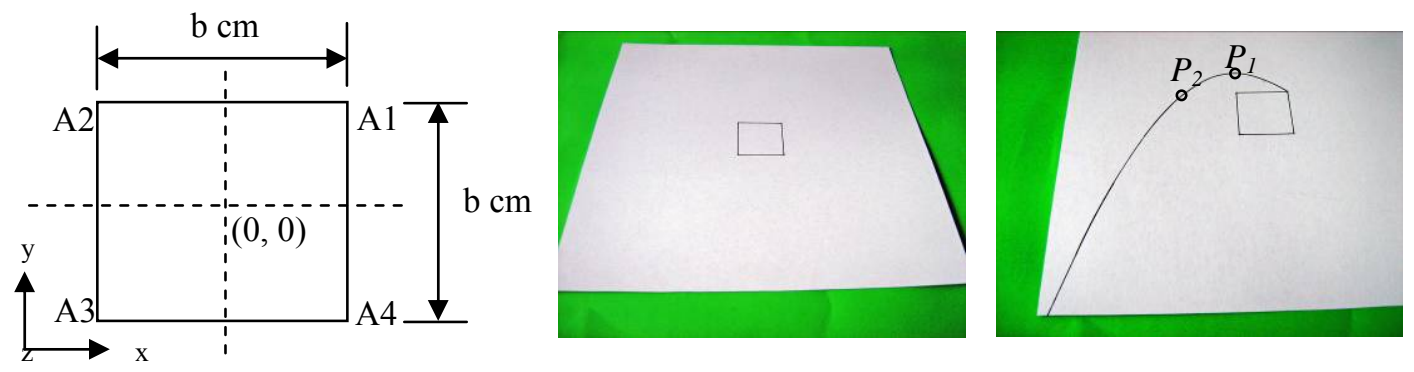

Figure 2: Construction of center square on the cardboard and first parabolic curve.

the first curve has been drawn, the remaining three curves are obtained through corresponding translation and rotation of $90^{\circ}$ (second curve), $180^{\circ}$ (third curve) and $270^{\circ}$ (fourth curve) respectively around the origin $(0,0)$ as shown in Figure $3 a$. Two models of SSCFL have been folded using $B=30 \mathrm{~cm}$ and $b=4 \mathrm{~cm}$ where the following set of two parabolic equations have been adopted for the curved fold lines:

$$
\begin{aligned}
& y=-\frac{1}{8}(x-2)^{2}+4 \\
& y=-\frac{1}{56}\left(11 x^{2}+42 x-240\right)
\end{aligned}
$$

The resulting surfaces are shown in Figure 3(b) (SSCFL1) and 3(c) (SSCFL2), respectively. It can be seen that each folded surface consists of two valley lines and two ridge lines. Figure 4 shows the sketch of orthogonal projection of SSCFL1 and SSCFL2 onto a horizontal plane where the two valley lines (V1, V2) and two ridge lines (R1, R2) are indicated.

For the purpose of computer generation, z-coordinates of the construction lines are then specified. The heights of center square points $\mathrm{A} 1, \mathrm{~A} 2, \mathrm{~A} 3$ and $\mathrm{A} 4$ has been taken as $10 \%$ of the plan dimension $\mathrm{B},(30 \mathrm{~cm} \times 10 \%=$ 


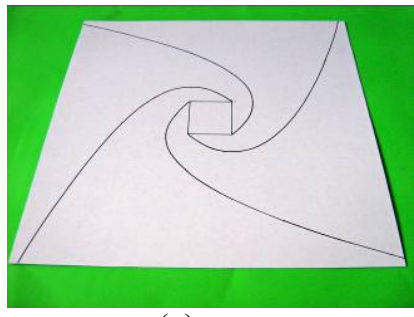

(a)

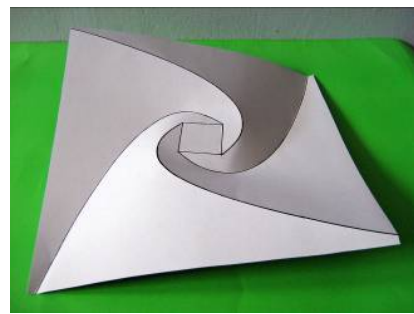

(b)

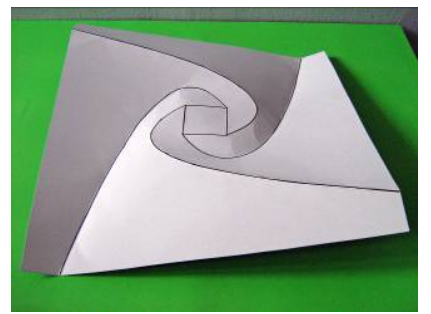

(c)

Figure 3: a) construction of second, third and fourth parabolic curve, b) SSFCL 1 after folding,

c) SSCFL 2 after folding

$3 \mathrm{~cm}$ ). Variation in height of curves V1 and V2 is chosen to be linear towards the edge. The height of points at the end of curves V1 and V2 is 0 . The ridge lines R1 and R2 have also been chosen to be varying linearly in height from the central square towards the edge. Height of the points at the end of ridge lines R1 and R2 is set to

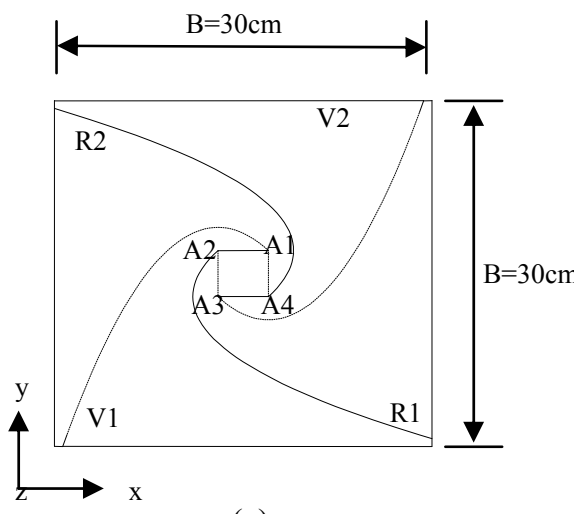

(a)

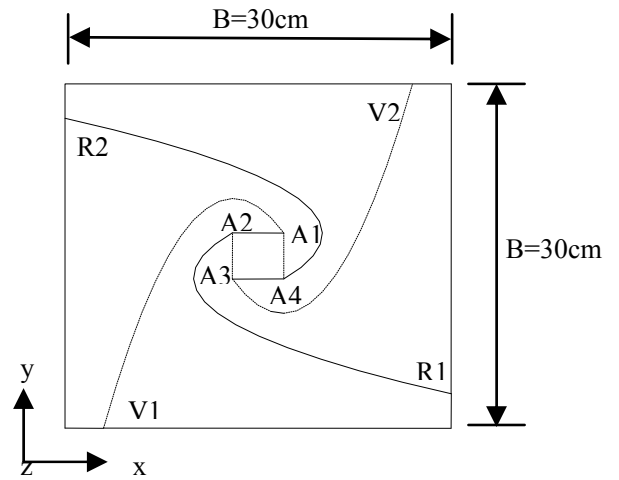

(b)

Figure 4: Construction of SSCFL on x-y plane, a) SSCFL 1, b) SSCFL 2

be $20 \%$ of plan dimension $\mathrm{B}(=0.2 \times 30 \mathrm{~cm}=6 \mathrm{~cm})$. The set of $(\mathrm{x}, \mathrm{y}, \mathrm{z})$ coordinates for the ridge and valley lines are transferred to computer program for the purpose of SSCFL surface generation. It is noted that other height variation of ridge and valley lines could also be used.

\subsection{Computer generation of SSCFL surface}

The following two main steps are involved in the generation of SSCFL model : i. creation of valley and ridge lines and ii. meshing of the four sub-surfaces defined by a pair of ridge-valley lines. Figure 5 shows the process. The generated model corresponding to SSCFL1 and SSCFL2 are shown in Figure 6.

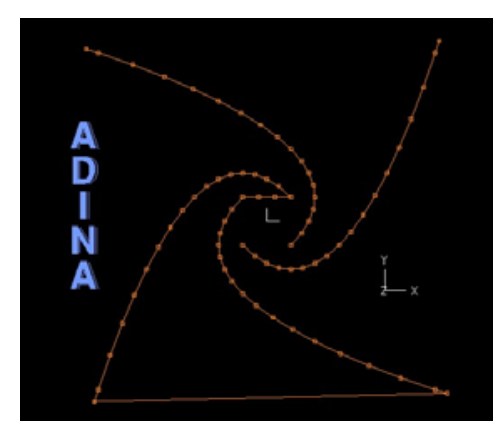

(a)

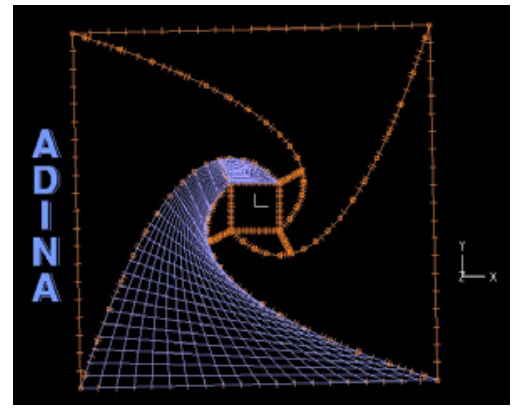

(b)

Figure 5: Main procedures in computer generation of SSCFL model : (a) specification of valley and ridge lines, (b) meshing of sub-surface defined by ridge-valley line pair 


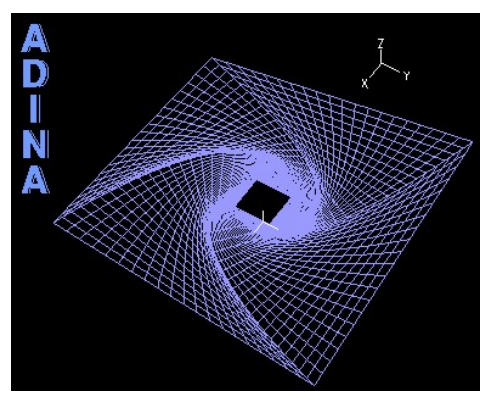

(a)

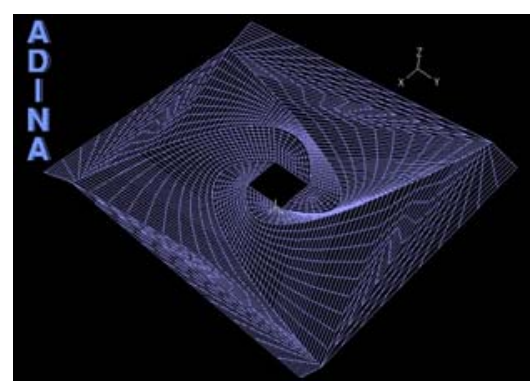

(b)

Figure 6: Computer model of: (a) SSCFL1 and (b) SSCFL2

\section{Discussion}

Folding process of SSCFL has revealed that surface form of SSCFL is arbitrary as it is influenced by the type of paper used, "sharpness" of the folds lines, condition along the edge (e.g.existence of fixed points) etc. As for the computer model, it is found that meshing of the sub-surface formed by pair of ridge-valley line will encounter difficulty if the curvature of fold lines surrounding the central square is too small. Such situation coupled with small central square relative to the plan size will result in "overlapping" of surface (where turn-back of surface occurs) if meshing is not carried out with care. Such problem could be overcome if each sub-surface is further sub-divided into smaller portions before meshing is carried out.

\section{Conclusion}

The idea of a novel structural form called shell surface with curved fold lines(SSCFL) has been introduced. Procedures for folding and generating SSCFL are presented. Further study in order : i. to investigate the effects of different versions of curved fold lines, plan-shapes, height variation of folds lines etc on the appearance of SSCFL and ii. to formulate an effective procedures for computer generation of SSCFL, are needed. Structural analysis will also need to be carried out on SSCFL models for the investigating of their structural behaviours.

\section{Acknowledgement}

Funding provided by Universiti Teknologi MARA (to the first author) and Universiti Sains Malaysia (to the second author) for the purpose of this research is highly appreciated.

\section{References}

[1] A.Razzack SA. A computational study on a nature inspired novel folded shell structure form. PhD Dissertation, Universiti Sains Malaysia, 2007.

[2] Harun MH. Analysis of shell surface with fold based on the idea from paper folding art. B Eng. Dissertation. Universiti Sains Malaysia, 2006

[3] Huffman DA. Curvature and Creases: A Primer on paper. IEEE Transactions On Computers, Vol. C-25, No. 10, October 1976; 1010-1019.

[4] Huffman DA. Geometric paper folding by Huffman: 1996. http://www. graficaobscura. com/Huffman/ index. html, excessed 2 January 2008.

[5] Jay L. How origami helps science, save lives. http://www.newsfactor.com/perl/story/16379.html. excessed 2 April 2008.

[6] Ketchum M. Concrete Shell Structures - Design, Analysis, \& Construction http://www.ketchum.org/ shells.html (accessed 2 January 2008)

[7] MD Resadi MKA. Shell surface with folds based on idea from paper folding art. B Eng. Dissertation. Universiti Sains Malaysia, 2007.

[8] Wartheim M. Cones, Curves, Shells, Towers: He Made Paper Jump to Life. The New York Times. Published June 22, 2004. http:// www.nytimes.com/2004/06/22/science/22orig.html?pagewanted=1\&ei= 5007\&en=48e57540cc74481 f\&ex=1403236800\&partner=USERLAND. (accessed 2 January 2008) 


\title{
Modeling of clothing and interactions with the body using continuum degenerated shell finite elements
}

\author{
Colby C. SWAN*, Xiaolin MAN, Rob W. WILLIAMS \\ *Department of Civil \& Environmental Engineering, The University of Iowa \\ 4120 Seamans Center, Iowa City, Iowa 52242 USA \\ Email: colby-swan@uiowa.edu
}

\begin{abstract}
A modeling framework is presented to assess the performance characteristics of protective clothing systems of various fabrics and cuts when interacting with moving human bodies in a virtual environment. While protective garments are worn to shield the wearer from a variety of threats such as ballistics, toxins, and extreme temperatures, they must also permit the wearer to perform all necessary job functions. The term performance characterization is used to denote how the garment affects the mobility and mechanical energy expenditure of the wearer. The clothing is modeled here using continuum degenerated shell finite elements with an anisotropic elastoplastic constitutive model. Realistic modeling of the human anthropometry is achieved with polygonal meshes obtained from laser body scans. Motion of the human body is prescribed and the clothing response is computed using explicit dynamics and frictional contact.
\end{abstract}

\section{Shell Framework}

In the framework being presented, the woven fabric that comprises the clothing is modeled using a continuum shell theory. The nonlinear, anisotropic, hysteretic constitutive behavior of clothing fabrics is modeled here using an anisotropic quadratic plasticity model with kinematic hardening. Within the continuum shell theory, the initial and updated domains occupied by a specific finite element of the clothing are, respectively:

$$
\mathbf{X}(\xi, \eta, \zeta)=\sum_{A=1}^{n e n} N_{A}(\xi, \eta)\left[\overline{\mathbf{X}}_{A}+z_{A}(\zeta) \hat{\mathbf{X}}_{A}\right] \quad \mathbf{x}(\xi, \eta, \zeta)=\sum_{A=1}^{n e n} N_{A}(\xi, \eta)\left[\overline{\mathbf{x}}_{A}+z_{A}(\zeta) \hat{\mathbf{x}}_{A}\right]
$$

Above $\overline{\mathbf{X}}_{A}$ and $\overline{\mathbf{x}}_{A}$ are the initial and updated reference surface coordinates; $\hat{\mathbf{X}}_{A}$ and $\hat{\mathbf{x}}_{A}$ are the initial and updated fiber directors; $z_{A}(\zeta)$ is a thickness function, and $N_{A}(\xi, \eta)$ is a two dimensional iso-parametric shape function associated with node $A$ and nen is the number of element nodes. This shell element has five nodal degrees of freedom: three translational components in a Cartesian coordinate system and two rotations about the in-plane nodal fiber directors (Fig. 1a). This traditional shell formulation does not explicitly account for through the thickness stretch and accordingly material models employed in such a framework must be projected into a shell stress sub-space in which the shell normal stress vanishes and the through-thickness stretch is computed at integration points in order to satisfy this constraint (Swan and Cakmak [8]). Alternative continuum shell theories that explicitly account for through-thickness stretch have been put forward by El-Abbasi and Meguid [3] and Cardoso and Yoon [2]. Although such elements have seven as opposed to five nodal degrees of freedom, incorporation of general nonlinear material models into such a framework is much more straightforward.

The displacement field within a 5-nodal-dof shell element is as follows:

$$
\mathbf{u}(\xi, \eta, \zeta)=\sum_{A=1}^{n e n} N_{A}(\xi, \eta)\left[\left(\overline{\mathbf{X}}_{A}-\overline{\mathbf{x}}_{A}\right)+z_{A}(\zeta)\left(\hat{\mathbf{x}}_{A}-\hat{\mathbf{X}}_{A}\right)\right]
$$


The normalized fiber director is updated by the rotational degrees of freedom $\theta_{1}$ and $\theta_{2}$ as follows:

$$
\hat{\mathbf{x}}_{A}=\hat{\mathbf{X}}_{A}+\frac{\sin \theta}{\theta}\left(\theta_{A 2}^{f} \mathbf{e}_{A 1}^{f}-\theta_{A 1}^{f} \mathbf{e}_{A 2}^{f}\right)+(1-\cos \theta) \mathbf{e}_{A 3}^{f} \quad \text { where } \theta=\sqrt{\boldsymbol{\theta} \cdot \boldsymbol{\theta}}
$$

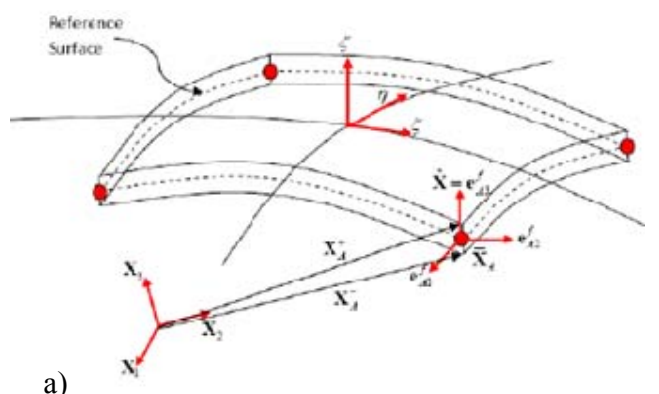

b)

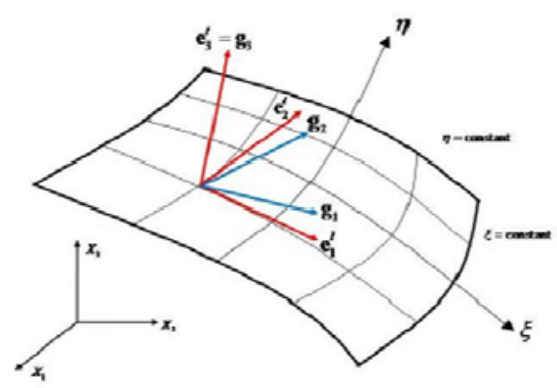

Fig. 1. a) geometry of shell element with reference surface and nodal fiber basis; b) material directors $\mathbf{g}_{1}$ and $\mathbf{g}_{2}$ that track fabric yarn orientations.

At a given time value $t_{n+1}$, the deformation gradient $\mathbf{F}_{n+1}$ is computed at the integration points of the shell elements with respect to the lamina coordinate system shown in Fig. 1b. The incremental deformation gradient for the $(\mathrm{n}+1)^{\text {th }}$ time step is computed $\mathbf{f}_{n+1}=\mathbf{F}_{n+1} \cdot \mathbf{F}_{n}^{-1}$ and used to update the material directors $\mathbf{g}^{1}$ and $\mathbf{g}^{2}$ from step $\mathrm{n}$ to those at step $\mathrm{n}+1$ :

$$
\left(\mathbf{g}^{i}\right)_{n+1}=\left(\frac{\partial \xi_{i}}{\partial x_{j}}\right)_{n+1} \mathbf{e}_{j}=\left(Q_{i j}\right)_{n+1}=\mathbf{f}_{n+1} \cdot\left(\mathbf{g}^{i}\right)_{n}
$$

The incremental Eulerian strain is computed as $\mathbf{e}_{n+1}=\frac{1}{2}\left(\mathbf{1}-\mathbf{f}_{n+1}^{-T} \cdot \mathbf{f}_{n+1}^{-1}\right)$ and the previous stresses and back-stresses, $\boldsymbol{\tau}_{\mathrm{n}}$ and $\boldsymbol{q}_{\mathrm{n}}$, the current strain, $\boldsymbol{e}_{\mathrm{n}+1}$, and the elastic moduli, $\mathbf{C}_{\mathrm{n}+1}$ are transformed from the element's lamina coordinate system into the fabric's current local convected coordinate system as follows:

$$
\widetilde{\boldsymbol{\tau}}_{n}=\mathbf{Q}_{n+1} \boldsymbol{\tau}_{n} \mathbf{Q}_{n+1}^{T} ; \quad \widetilde{\mathbf{q}}_{n}=\mathbf{Q}_{n+1} \mathbf{q}_{n} \mathbf{Q}_{n+1}^{T} ; \quad \widetilde{\mathbf{e}}_{n+1}=\mathbf{Q}_{n+1}^{-1} \mathbf{e}_{n+1} \mathbf{Q}_{n+1}^{-T} ; \quad \widetilde{\mathbf{C}}_{n+1}=\mathbf{Q}_{n+1}^{T} \mathbf{Q}_{n+1} \mathbf{C}_{n} \mathbf{Q}_{n+1}^{T} \mathbf{Q}_{n+1} ;
$$

Once the elastic stress predictors are computed as follows

$$
\widetilde{\boldsymbol{\tau}}_{n+1}^{t r}=\widetilde{\boldsymbol{\tau}}_{n}+\widetilde{\mathbf{C}}_{n+1}: \widetilde{\mathbf{e}}_{n+1} ; \quad \widetilde{\mathbf{q}}_{n+1}^{t r}=\widetilde{\mathbf{q}}_{n} ; \quad \widetilde{\boldsymbol{\eta}}_{n+1}^{t r}=\widetilde{\boldsymbol{\tau}}_{n}-\widetilde{\mathbf{q}}_{n+1}^{t r}
$$

the yield function is evaluated as follows:

$$
\widetilde{\phi}_{n+1}^{t r}=\widetilde{\eta}_{n+1}^{t r}: \overline{\mathbf{A}}: \widetilde{\eta}_{n+1}^{t r}-1
$$

When $\widetilde{\phi}_{n+1}^{t r}>0$, an implicit closest point projection return map is performed as described in [3]. Once the stress $\widetilde{\boldsymbol{\tau}}_{n+1}$, and back-stress $\widetilde{\mathbf{q}}_{n+1}$ are updated in the return map algorithm, they are then rotated back into the shell element's lamina coordinate system and used to compute element forces and stiffness terms. In Eq. (7), $\overline{\mathbf{A}}_{n+1}$ contains the squared inverted radii of the elastic domain in stress space which are chosen to reflect the directional strength properties of the fabric measured experimentally (Fig. 2).

\section{A Dynamic Virtual Mannequin}

The starting point for a virtual mannequin is a laser body scan of a human subject. For this effort, co-author XM was scanned in the facility of Prof. Susan Ashdown at Cornell University [1]. Typical body scans yield discretizations of the human body surface involving on the order of hundreds of thousands of nodes and polygons. Such meshes are much finer than is necessary, and indeed usage of such fine meshes would be prohibitively expensive computationally in clothing contact modeling. Accordingly, the body-surface mesh can 
be coarsened using commercial software tools. In Fig. 3 the original body-surface scan is shown together with a coarsened scan involving less than $1 \%$ of the original number of nodes and polygons.

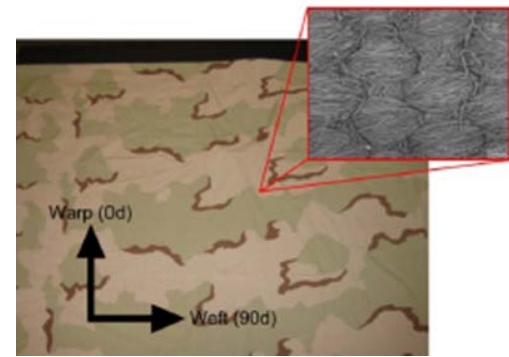

a) b)

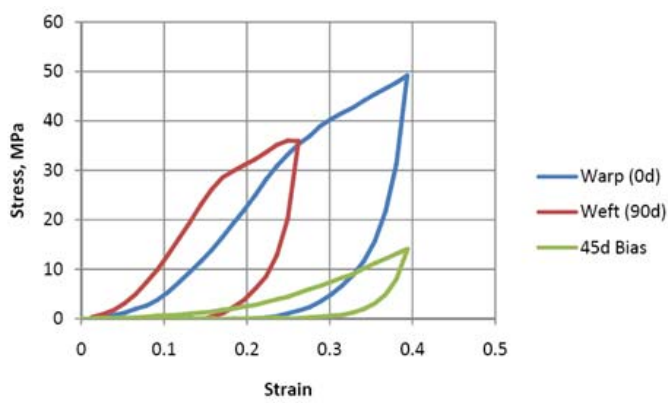

Fig. 2. a) plain-weave cotton fabric with warp $\left(0^{\circ}\right)$ and weft $\left(90^{\circ}\right)$ directions; and b) representative measured stress-strain responses of the fabric in the $0^{\circ}, 90^{\circ}$, and $45^{\circ}$ directions.

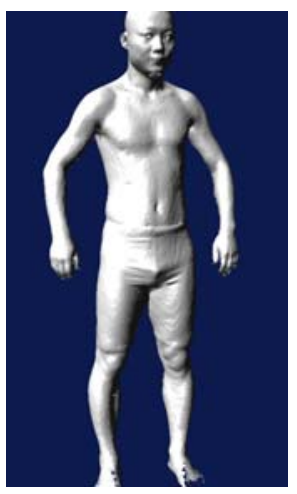

(a)

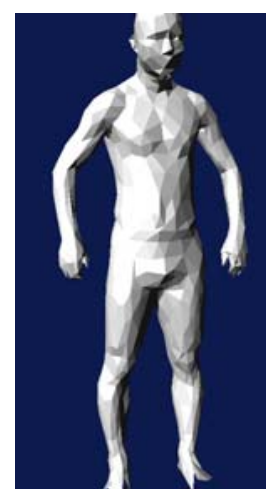

(b)

Fig.3. Original body scan mesh (a) of $\mathbf{2 3 0 , 0 0 0}$ polygons and significantly coarsened mesh (b) of only 1,700 polygons.

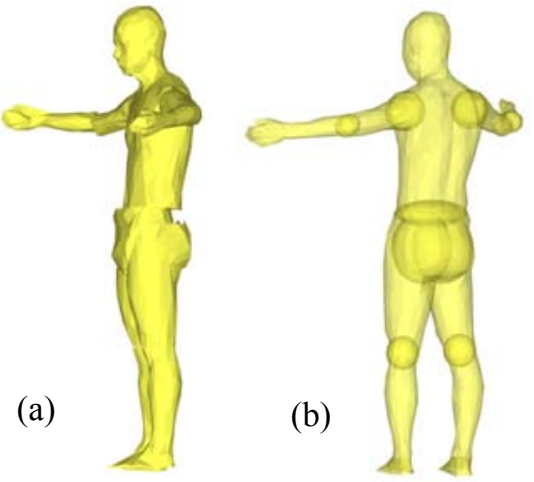

Fig. 4. Dynamic virtual mannequin in two postures during activity driven by motioncaptured data. (a) without spheres and ellipsoids at joints, discontinuities develop; and (b) with spheres and ellipsoids, smoothness at joints.

Once a complete and coarsened body scan mesh is available, it is decomposed into an assemblage of individual meshes corresponding to limb, torso, and appendage segments. The optimal decomposition of meshes into segments can itself be quite involved (Lien [5]) but was done here in an ad-hoc manner at the primary joint locations (Rasmussen [7]). To represent the human body during activity, each segment of the body-surface mesh is driven in space and time as a rigid-body using motion-capture data of the human subject performing a representative exercise (Fig. 4) or using predictive human dynamics models such as those of Kim et al [4]. The body-surface mesh is smoothed at the joints using spheres and ellipsoids to preclude development of gaps and holes in the mesh that would otherwise occur. In this manner, a dynamic virtual mannequin is obtained as a set of master surfaces with which the clothing model will undergo unilateral contact.

\section{Collision Detection and Contact Computation}

The discrete equations of motion for the clothing are solved using an explicit time-integration scheme. Stability is maintained by using a time step smaller than that needed to maintain Courant stability. The clothing motion is driven by gravitational body forces and frictional contact with the virtual mannequin. In the present analysis model the motion of the virtual mannequin is fully prescribed and the resulting force time histories that the clothing exerts on the body are computed. The impenetrability constraint between the clothing model and a virtual mannequin is enforced using an explicit master-slave frictional contact algorithm. In particular 
mannequin penetrations of the clothing are detected and corrected, as are clothing penetrations of the mannequin. In the present framework, clothing self-penetrations are neither detected nor corrected.

\section{Results}

Previous finite element models of mechanical interactions between the human body and clothing were presented by Man and Swan [6] in which the human body was modeled as an assemblage of ellipsoids. Here the body surface is modeled with polygonal meshes obtained from body scans, and the clothing model consists of pants and a shirt made of plain-weave cotton fabric. To get the clothing model onto the mannequin, the two are first co-located without regard to penetrations. Once co-located, gravity loading is turned on as is the contact algorithm yielding the clothing model donned on the mannequin (Fig. 5a). From this stage, the mannequin can then begin to move and the clothing response computed using explicit dynamics and contact (Fig. 5b).
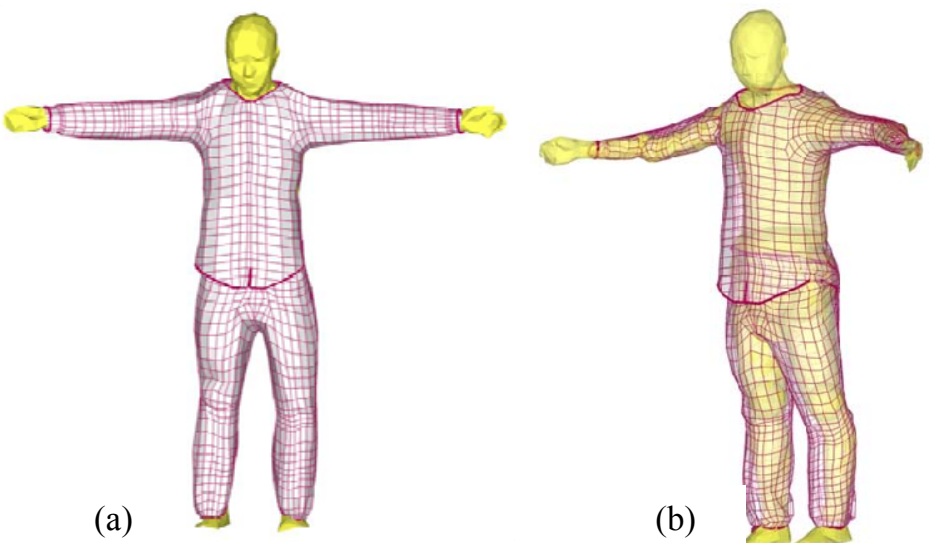

Fig. 5. (a) clothing model donned on the virtual mannequin in fixed initial posture; (b) clothing model moving with and responding to the mannequin

\section{Acknowledgements}

This research was funded under grants from the U.S. Army Soldier Systems Center. This support is gratefully acknowledged.

\section{References}

[1] Ashdown S www.bodyscan.human.cornell.edu 2006.

[2] Cardoso, RPR and Whan YJ One point quadrature shell element with through thickness stretch. Computer Methods in Applied Mechanics and Engineering, 2005;194(9-11): 1161-99.

[3] El-Abbasi, N. and Meguid, SA. A new shell element accounting for through-thickness deformation. Computer Methods in Applied Mechanics and Engineering, 2000; 189(3): 841-62.

[4] Kim H, Wang Q, Rahmatalla S, Swan CC, Arora JS, Malek KA and Assouline JG, Dynamic motion planning of 3D human locomotion using gradient-based optimization, J. Biomechanical Engineering 2008; In press.

[5] Lien J-M. Approximate convex decomposition and its applications, PhD Dissertation, Texas A\&M University (2006).

[6] Man X and Swan CC A mathematical modeling framework for analysis of functional clothing, Journal of Engineered Fibers and Fabrics, 2007;2(3).

[7] Rasmussen, MK An analytical framework for the computational modeling of virtual clothing-mannequin interaction using finite element analysis, MS Thesis, The University of Iowa, 2008.

[8] Swan CC and Cakmak AS A hardening orthotropic plasticity model for pressure-insensitive composites: continuum formulation and integration algorithm, International Journal for Numerical Methods in Engineering., 37 839-60 (1994). 


\title{
Structural analysis for multi-folding and deployable structures
}

\author{
Masatoshi NAKAZAWA, Ichiro ARIO*, Andrew WATSON \\ * Dept. of Civil \& Environmental Eng., Hiroshima University \\ Higashi-Hiroshima, 739-8527, Japan \\ E-mail: mario@hiroshima-u.ac.jp
}

\begin{abstract}
In order to develop the energy absorption device such as folding truss, we consider the pantograph structure with allowing for plastic deformation. The folding behaviour of a pantograph is complicated by the unsymmetric snap-through behaviour not only symmetric mode under the compressive loading. If the compressive loading is substantially large, plastic deformation occurs in the member and causes the stress re-distribution of structures. This paper presents the analysis for such structure expressing the plastic behaviour prior to snap-through behaviour. Unsymmetric postbuckling behaviour and the elasto-plastic behaviour must be made clear to evaluate the performance of this type of smart structures.
\end{abstract}

\section{Introduction}

Recently, Holnicki-Szulc et al. [2, 3] proposed a new concept for energy absorption as a smart structure. This concept was based upon his experiments where he demonstrated the elastic folding behaviour of a pantograph subject to compressive loading.

In the multi-folding microstruture(MFM) of a pantograph, the buckling or snap-through will occur in the compressed uniaxial members and loses its stiffness suddenly. Hence, the absorption of impact energy is achieved by the bifurcation behaviour of uni-axial member. Ario and Watson[1] formulated this bifurcation phenomena analytically, and investigated the elastic behaviour by the dynamic analysis.

However, in the experimental study, the control of buckling behaviour of uniaxial members is difficult, and it is general to use the controllable stiffness for uni-axial direction. Hence, the longitudinal stiffness is likely to limited intentionally by the induced stress. This is the same principle as the plastification of material, because that the member loses its stiffness and the deformation flows. Moreover, unsymmetric snap-through behaviour is also found in the experiments, and it is important to make clear the mechanism of occurrence.

This paper presents the elasto-plastic behaviour of the multi-folding microstructure by dynamic analysis. The difference in behaviour are compared with elastic ones and it can lead to a more efficient design. The analytical equation is formulated for multi-layer of pantograph structures as shown in Fig. 1 to generalize the analytical procedure of solution.

\section{Analytical equation and solution of MFM for elastic behaviour}

The generalized pantograph structures shown in Fig. 1(a) has right-left symmetry. However, in order to consider the unsymmetric deformation, the full model is formulated here. Now, we assume a periodic height for each layer of $h_{i}=\gamma_{i} L$ where the width of truss structure $L$ is fixed. Hence, the initial length of each bar is expressed as 


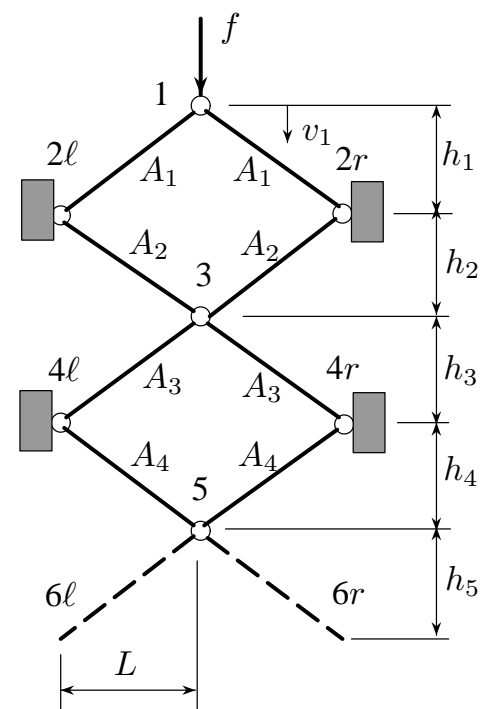

(a) generalized pantograph structures

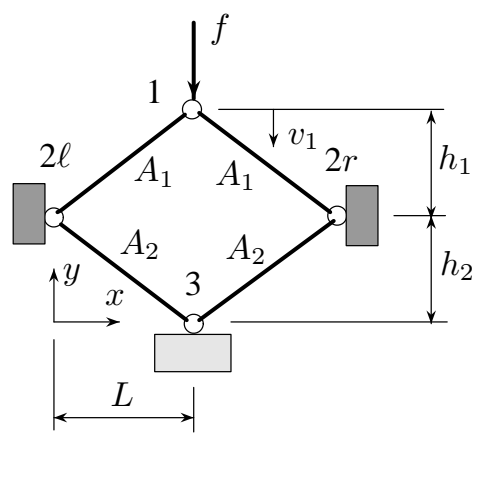

(b) diamond-shape truss

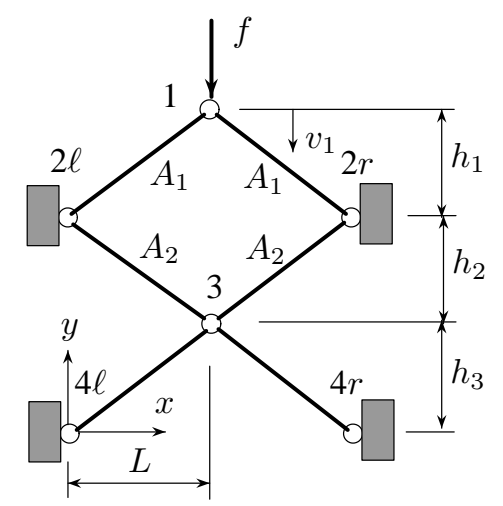

(c) 3 bar truss

Figure 1: Generalized pantograph structures

$\ell_{i}=\sqrt{L^{2}+h_{i}^{2}}=L \sqrt{1+\gamma_{i}^{2}},(i=1,2, \cdots, n)$ where $n$ is the number of layer. The length $\hat{\ell}_{i, k},(k=\ell, r)$ after deformation is a function of height and nodal displacement such as

$$
\begin{aligned}
\hat{\ell}_{1, \ell} & =\sqrt{L^{2}+\left\{\left(h_{1}+h_{2}+\cdots+h_{n}-v_{1}\right)-\left(h_{2}+\cdots+h_{n}-v_{2, \ell}\right)\right\}^{2}}=L \sqrt{1+\left(\gamma_{1}-\bar{v}_{1}+\bar{v}_{2, \ell}\right)^{2}} \\
\hat{\ell}_{2, \ell} & =L \sqrt{1+\left(\gamma_{2}-\bar{v}_{2, \ell}+\bar{v}_{3}\right)^{2}}, \quad \hat{\ell}_{2, r}=L \sqrt{1+\left(\gamma_{2}-\bar{v}_{2, r}+\bar{v}_{3}\right)^{2}} \\
\therefore \hat{\ell}_{i, k} & =L \sqrt{1+\left(\gamma_{i}-\bar{v}_{i, k}+\bar{v}_{i+1, k}\right)^{2}}, \quad(k=\ell, r)
\end{aligned}
$$

where $\gamma_{i}=h_{i} / L$ and $\hat{v}_{i}=v_{i} / L,(i=1,2, \cdots, n) . \ell$ and $r$ means left and right, respectively, and note that even node has two nodes of left and right. Using Green's strain expression, we obtain the strain in each truss bar as

$$
\varepsilon_{i} \equiv \frac{1}{2}\left\{\left(\frac{\hat{\ell}_{i}}{\ell_{i}}\right)^{2}-1\right\}=\frac{1}{2}\left\{\frac{1+\left(\gamma_{i}-\bar{v}_{i}+\bar{v}_{i+1}\right)^{2}}{1+\gamma_{i}^{2}}-1\right\}, \quad(i=1,2, \cdots, n)
$$

From above, the total potential energy of this system is given by

$$
U \equiv \sum_{i=1}^{n} \frac{E A_{i}}{2}\left(\varepsilon_{i, \ell}^{2}+\varepsilon_{i, r}^{2}\right)-f \bar{v}_{1} L=\sum_{i=1}^{n} \frac{\beta_{i} L}{8}\left\{q_{i, \ell}^{2}\left(q_{i, \ell}-2 \gamma_{i}\right)^{2}+q_{i, r}^{2}\left(q_{i, r}-2 \gamma_{i}\right)^{2}\right\}-f \bar{v}_{1} L
$$

where $f$ is a concentrated load at node $1, \beta_{i}=E A_{i} /\left(1+\gamma_{i}^{2}\right)^{3 / 2}$, and $q_{i, k}=\bar{v}_{i, k}-\bar{v}_{i+1, k}, \quad(k=\ell, r)$.

In the case of 2 layer pantograph structure, namely diamond-shape folding system as shown in Fig. 1(b), the analytical solution of load-deflection relationships considering the unsymmetric deformation can be obtained. Total potential energy of 2 layer pantograph system is given by

$$
U=\frac{L}{8}\left[\beta_{1}\left\{q_{1, \ell}^{2}\left(q_{1, \ell}-2 \gamma_{1}\right)^{2}+q_{1, r}^{2}\left(q_{1, r}-2 \gamma_{1}\right)^{2}\right\}+\beta_{2}\left\{q_{2, \ell}^{2}\left(q_{2, \ell}-2 \gamma_{2}\right)^{2}+q_{2, r}^{2}\left(q_{2, r}-2 \gamma_{2}\right)^{2}\right\}\right]-f \bar{v}_{1} L
$$




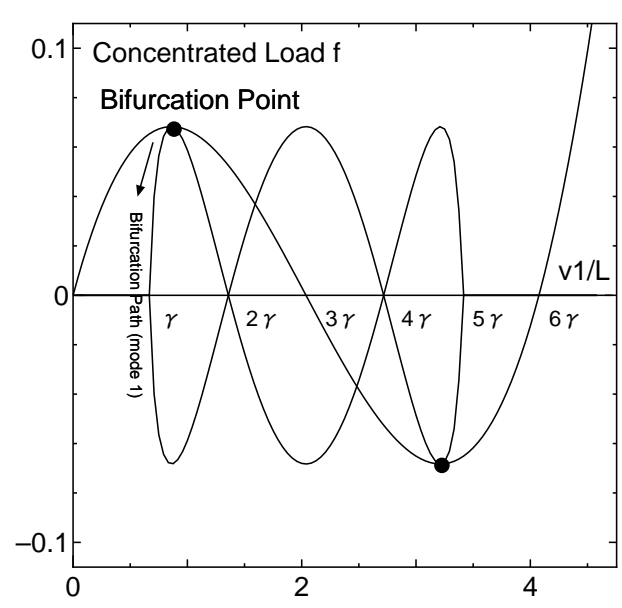

Figure 2: Analytical solution

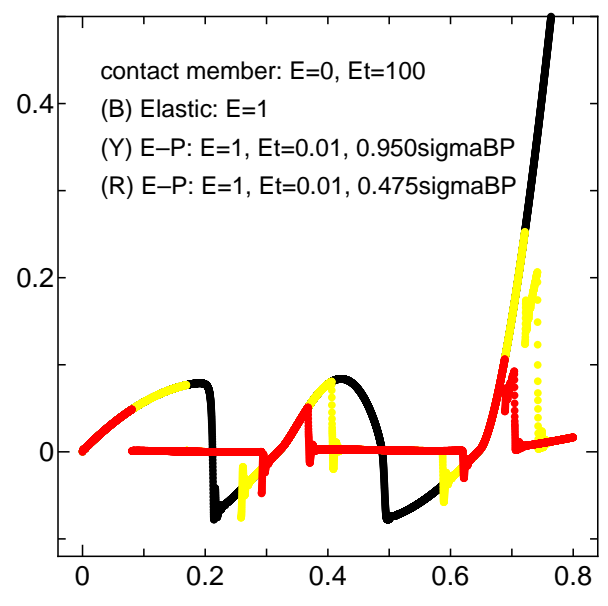

Figure 3: Elasto-plastic behaviour for mode 1(top member initiating folding pattern)

and equilibrium equations are derived by stationary condition as,

$$
\begin{gathered}
F_{1}=\frac{\partial U}{\partial v_{1}}=0 \quad: \quad \frac{\beta_{1}}{2}\left\{q_{1, \ell}\left(q_{1, \ell}-\gamma_{1}\right)\left(q_{1, \ell}-2 \gamma_{1}\right)+q_{1, r}\left(q_{1, r}-\gamma_{1}\right)\left(q_{1, r}-2 \gamma_{1}\right)\right\}-f=0 \\
F_{2, \ell}=\frac{\partial U}{\partial v_{2, \ell}}=0 \quad: \quad-\beta_{1} q_{1, \ell}\left(q_{1, \ell}-\gamma_{1}\right)\left(q_{1, \ell}-2 \gamma_{1}\right)+\beta_{2} \bar{v}_{2, \ell}\left(\bar{v}_{2, \ell}-\gamma_{2}\right)\left(\bar{v}_{2, \ell}-2 \gamma_{2}\right)=0 \\
F_{2, r}=\frac{\partial U}{\partial v_{2, r}}=0 \quad: \quad-\beta_{1} q_{1, r}\left(q_{1, r}-\gamma_{1}\right)\left(q_{1, r}-2 \gamma_{1}\right)+\beta_{2} \bar{v}_{2, r}\left(\bar{v}_{2, r}-\gamma_{2}\right)\left(\bar{v}_{2, r}-2 \gamma_{2}\right)=0 \\
\therefore \quad f=\frac{\beta_{1}}{2}\left\{q_{1, \ell}\left(q_{1, \ell}-\gamma_{1}\right)\left(q_{1, \ell}-2 \gamma_{1}\right)\right\}
\end{gathered}
$$

If we consider the simple model of $\beta_{i}=\beta$ and $\gamma_{i}=\gamma$, the load-deflection curve is expressed by

$$
\begin{gathered}
F_{2, r}=0 \quad: \quad-\left(\bar{v}_{1}-\bar{v}_{2, r}\right)\left(\bar{v}_{1}-\bar{v}_{2, r}-\gamma\right)\left(\bar{v}_{1}-\bar{v}_{2, r}-2 \gamma\right)+\bar{v}_{2, r}\left(\bar{v}_{2, r}-\gamma\right)\left(\bar{v}_{2, r}-2 \gamma\right)=0 \\
\therefore \quad \bar{v}_{2, r}=\frac{\bar{v}_{1}}{2}, \quad \bar{v}_{2, r}=\frac{1}{2}\left\{\bar{v}_{1} \pm \sqrt{\bar{v}_{1}^{2}-4\left(\bar{v}_{1}-\gamma\right)\left(\bar{v}_{1}-2 \gamma\right)}\right\} \\
F_{2, \ell}=0 \quad: \quad-\left(\bar{v}_{1}-\bar{v}_{2, \ell}\right)\left(\bar{v}_{1}-\bar{v}_{2, \ell}-\gamma\right)\left(\bar{v}_{1}-\bar{v}_{2, \ell}-2 \gamma\right)+\bar{v}_{2, \ell}\left(\bar{v}_{2, \ell}-\gamma\right)\left(\bar{v}_{2, \ell}-2 \gamma\right)=0 \\
\quad \therefore \quad \bar{v}_{2, \ell}=\frac{\bar{v}_{1}}{2}, \quad \bar{v}_{2, \ell}=\frac{1}{2}\left\{\bar{v}_{1} \pm \sqrt{\bar{v}_{1}^{2}-4\left(\bar{v}_{1}-\gamma\right)\left(\bar{v}_{1}-2 \gamma\right)}\right\} \\
F_{1}=0 \quad: \quad f=\frac{\beta}{2}\left\{\left(\bar{v}_{1}-\bar{v}_{2, \ell}\right)\left(\bar{v}_{1}-\bar{v}_{2, \ell}-\gamma\right)\left(\bar{v}_{1}-\bar{v}_{2, \ell}-2 \gamma\right)+\left(\bar{v}_{1}-\bar{v}_{2, r}\right)\left(\bar{v}_{1}-\bar{v}_{2, r}-\gamma\right)\left(\bar{v}_{1}-\bar{v}_{2, r}-2 \gamma\right)\right\}
\end{gathered}
$$

As a summary, two symmetric solutions are obtained as follows:

$$
\begin{array}{ll}
f=\frac{\beta}{8} \bar{v}_{1}\left(\bar{v}_{1}-2 \gamma\right)\left(\bar{v}_{1}-4 \gamma\right), \quad \text { for } \quad \bar{v}_{2, \ell}=\bar{v}_{2, r}=\frac{\bar{v}_{1}}{2} \\
f=-\beta\left(\bar{v}_{1}-\gamma\right)\left(\bar{v}_{1}-2 \gamma\right)\left(\bar{v}_{1}-3 \gamma\right), \quad \text { for } \quad \bar{v}_{2, \ell}=\bar{v}_{2, r}=\frac{1}{2}\left\{\bar{v}_{1} \pm \sqrt{\bar{v}_{1}^{2}-4\left(\bar{v}_{1}-\gamma\right)\left(\bar{v}_{1}-2 \gamma\right)}\right\}
\end{array}
$$

and moreover, unsymmetric solution is obtained as follows:

$$
\begin{aligned}
& f=-\beta\left(\bar{v}_{1}-\gamma\right)\left(\bar{v}_{1}-2 \gamma\right)\left(\bar{v}_{1}-3 \gamma\right) \\
& \quad \text { for } \quad \bar{v}_{2, \ell}=\frac{1}{2}\left\{\bar{v}_{1} \pm \sqrt{\bar{v}_{1}^{2}-4\left(\bar{v}_{1}-\gamma\right)\left(\bar{v}_{1}-2 \gamma\right)}\right\} \quad \text { and } \quad \bar{v}_{2, r}=\frac{1}{2}\left\{\bar{v}_{1} \mp \sqrt{\bar{v}_{1}^{2}-4\left(\bar{v}_{1}-\gamma\right)\left(\bar{v}_{1}-2 \gamma\right)}\right\}
\end{aligned}
$$

The extreme value of fundamental path is easily calculated as $f=\frac{2}{3 \sqrt{3}} \beta \gamma^{3}$ for $\bar{v}_{1}=\frac{2}{3}(3-\sqrt{3}) \gamma$, and these curves go through this point, namely this point is bifurcation point of primary path. It is also interesting that the symmetric and unsymmetric solution have the same load-deflection relationships. 


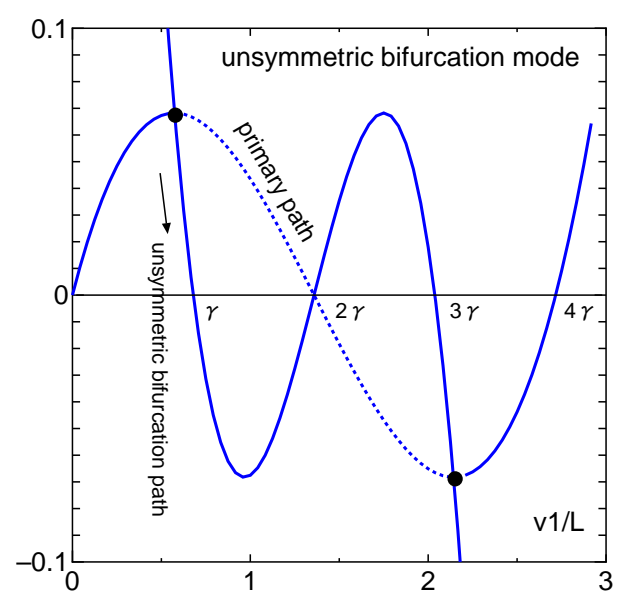

Figure 4: Analytical solution

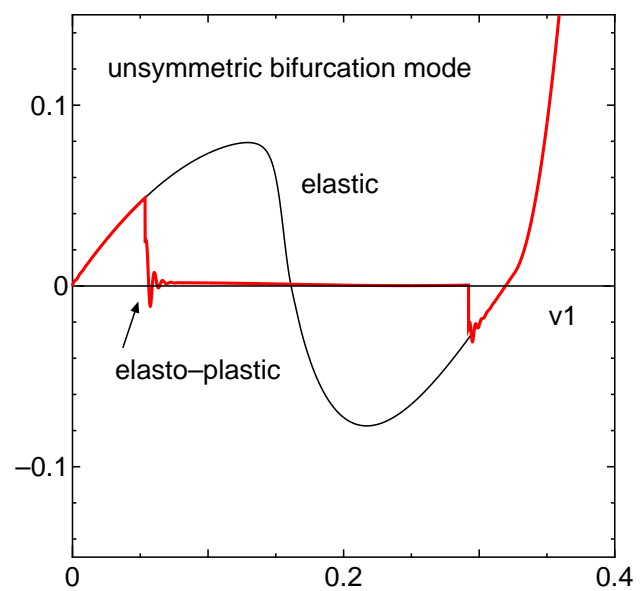

Figure 5: Elasto-plastic behaviour for unsymmetric mode of diamond-shape truss

\section{Folding characteristics of MFM allowing for elasto-plastic behaviour}

Elasto-plastic material property of bilinear type is given for the dynamic problems of 3 bar truss structures as shown in Fig. 1(c), and the results of load-displacement characteristics are summarized. The formulation of elastic behaviour is presented in Ref.[3], and bifurcation paths are presented in Fig. 2. Typical folding patterns of mode 1 for top member initiating folding are simulated, and the results are shown in Fig. 3. Three different yield stress level are given: i.e. (B) for fully elastic material, $0.95 \sigma_{Y}$ and $0.47 \sigma_{Y}$ for elasto-plastic material, where $\sigma_{Y}$ is the yield stress at which this material yields at bifurcation point simultaneously. The dynamic analysis for fully elastic material surely trace the load-displacement curve obtained by using the analytical solution and shows the snap-through from bifurcation point. For elasto-plastic material, the yielding of material precedes the bifurcation of the elastic materials, and also shows the snap-through behaviour at the yield point. Fig. 3 shows that only deviate from the elastic path at stress level exceeds the yield stress. Once the yielding occurs, the loading level becomes almost zero because of nearly zero stiffness. Hence, the overall load-displacement characteristics can be traced by the elastic analysis, although the influence of material yielding is recognized in early occurrence of snap-through. As a result, we can predict the general load-displacement behaviour of MFM structures by the elastic analysis.

\section{Unsymmetric postbuckling behaviour and elasto-plastic characteristics of diamond-shape truss}

The bifurcation paths obtained by analytical solution is presented in Fig. 4, and the corresponding numerical results by the dynamic and plastic analysis are also shown in Fig. 5. The critical truss member shows the yielding when the stress reaches to the yield stress, but post-yielding behaviour is stable. Once the yielding occurs, the loading level becomes almost zero because of smaller stiffness. Hence, the overall load-displacement characteristics also can be traced by the elastic analysis.

\section{References}

[1] Ario, I. and Watson, A. DYNAMIC FOLDING ANALYSIS FOR MULTI-FOLDING STRUCTURES UNDER IMPACT LOADING, Int. J. of Sound and Vibration, Vol.308/3-5, pp. 591-598, 2007.

[2] J. Holnicki-Szulc, P. Pawlowski and M. Wiklo High-performance impact absorbing materials - the concept, design tools and applications, Smart Materials and Structures, 12, pp. 461-467, 2003.

[3] J. Holnicki-Szulc and P. Pawlowski: The concept of multifolding and its experimental validation, the proceedings of XXI ICTAM, Warsaw, 2004. 


\title{
Structural behaviour of shell surface in the form of Möbius strip
}

\author{
$\underline{\text { Kok Keong CHOONG}}^{*}$, Min Sheng KUAN \\ *School of Civil Engineering, Universiti Sains Malaysia \\ 14300 Nibong Tebal, Penang, Malaysia \\ cekkc@eng.usm.my
}

\begin{abstract}
Thin shell surface in the form of Möbius strip is investigated with the objective of gaining basic understanding about its structural behaviour. Computational models of the surface are first generated and next analysed using finite element method under self-weight loading condition. Strength and stiffness of the surface are studied under the influence of different support conditions. Numerical results show that choice of support condition has a significant influence on the structural performance of the Möbius strip shell surface investigated.
\end{abstract}

\section{Introduction}

The Möbius strip is a one-sided non-orientable surface as shown in Figure 1. The form of Möbius strip has been

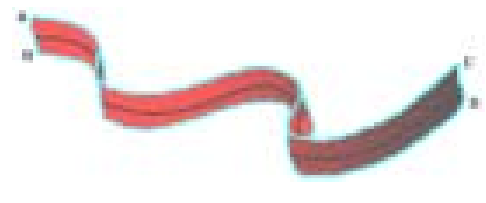

(a)

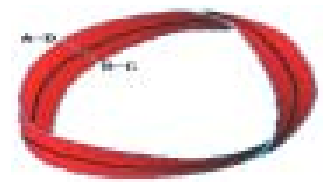

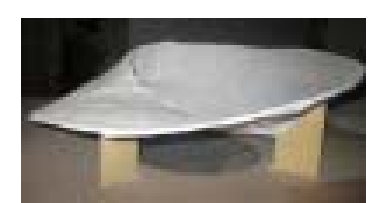

(b)

Figure 1: (a) Simple steps to produce a Möbius strip; (b) A model of Möbius strip

adopted in the creation of sculpture(Friedman, [1]), exploration of idea for bridges and for buildings(Séquin, [3])(Figure 2).
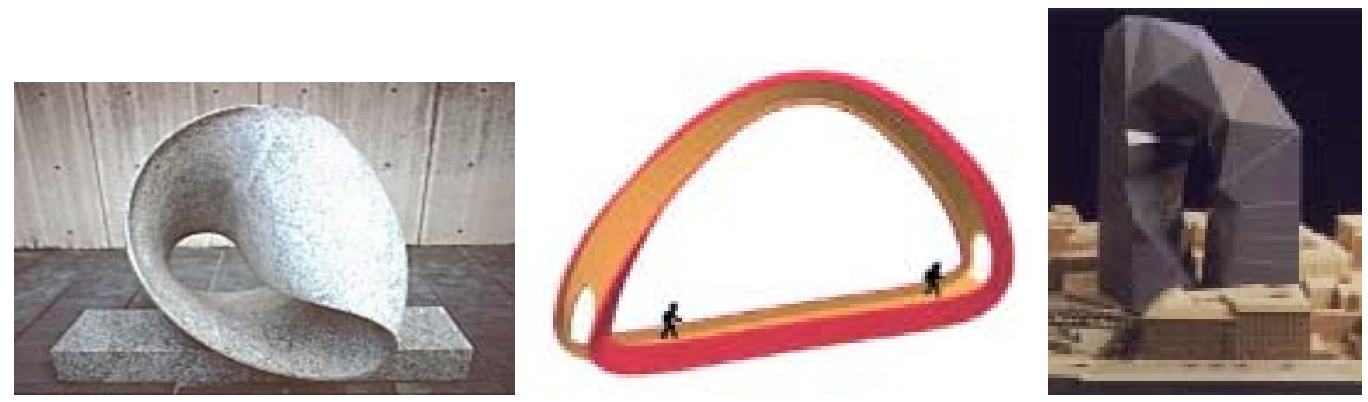

Figure 2: Möbius strip in: (a) granite sculpture, (b) idea for a bridge and (c) idea for a building

However, none of the examples mentioned present any results on the form of Möbius strip as load carrying members. One example closest to the idea of using the form of Möbius strip as load carrying members is the children playground as mentioned in Peterson[2] (see Figure 3). However such climber structure in children playground lacks the feature of being a continuous strip (see Figure 1) which is one of the principal reason why Möbius strip has generated so much interest. 


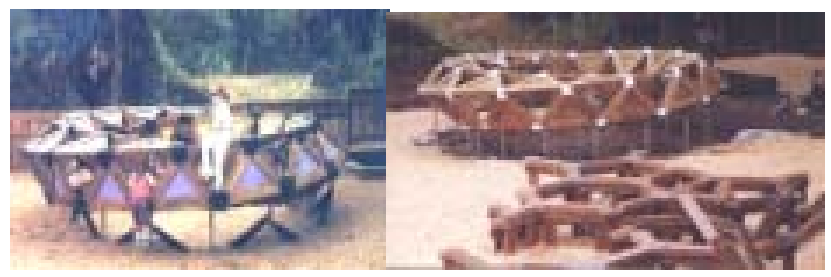

Figure 3: Climber at children playground modeled after the form of Möbius strip

This study is carried out with the objective of exploring the structural feasibility of adopting Möbius strip as the form for shell structure. Generation of Möbius strip shell surface is first described. This is then followed by numerical analysis carried out on model of Möbius strip shell surface generated under two different sets of boundary condition. Structural aspects of strength and stiffness are examined under self-weight condition.

\section{Generation of Möbius strip shell surface}

A Möbius strip of half-width $W$, with midcircle of radius $\mathrm{R}$ as shown in Figure 4, can be represented parametrically by the following set of equations(Weisstein, [4]):

$$
X=\left(R+S \cos \frac{\theta}{2}\right) \cos \theta, Y=\left(R+S \cos \frac{\theta}{2}\right) \sin \theta, Z=S \sin \left(\frac{\theta}{2}\right)
$$

for $\{S:-W, W\}$ and $\{\theta: 0,2 \pi\}$.

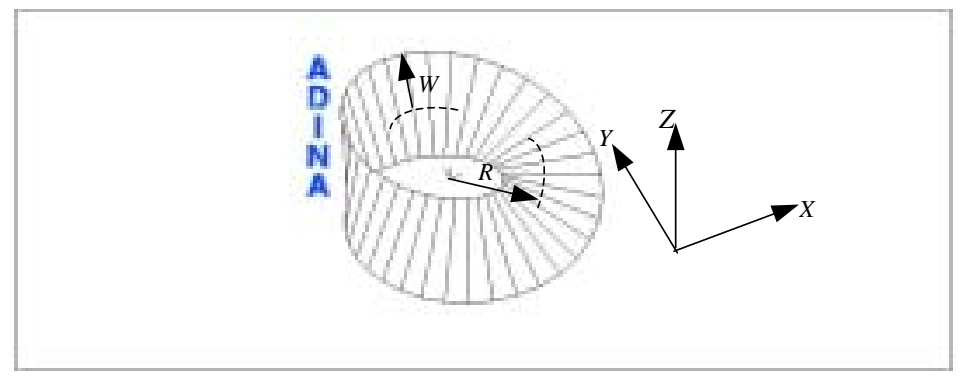

Figure 4: A Möbius strip with midcircle radius $R$ and half-width $W$

Taking into account the requirement of proper surface orientation in finite element(FE) analysis, generation of FE model has been carried out as shown in Figure 5.

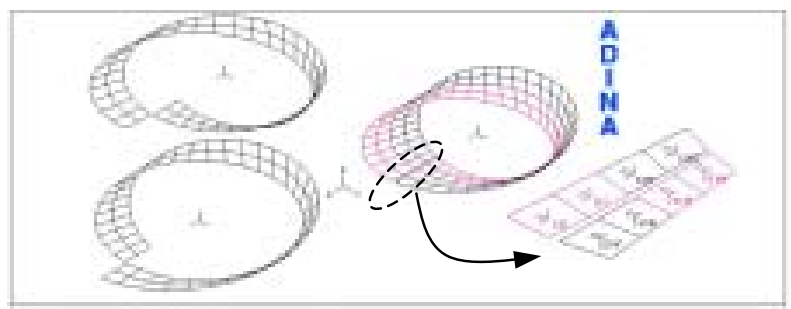

Figure 5: Generation procedures for the Möbius strip shell surface

Difficulty has been encountered in ensuring proper surface orientation of the Möbius strip shell surface. As a result, a small gap across the width of the strip has to be created in order to overcome the above mentioned problem as depicted in a detailed view in Figure 5.

\section{Numerical analysis}

The FE model generated $(R=10 \mathrm{~m}, W=3.5 \mathrm{~m})$ using 4-node isoparametric shell elements together with the two different sets of support conditions assigned (Case I and Case II) are shown in Figure 6. In Case I, all points 
along the central ring with radius $R$ which lies parallel to $x-y$ plane are hinged. As for Case II, the vertical part of the strip surface is fixed together with four points located on the ends of portion inclined at $45^{\circ}$ from horizontal plane hinged. Thickness of the shell surface is $t=350 \mathrm{~mm}$, material characteristics adopted is concrete with elastic modulus $\mathrm{E}=20 \mathrm{GPa}$, Poisson's ratio $v=0.15$ and self-weight $\gamma_{\mathrm{c}}=24 \mathrm{kN} / \mathrm{m}^{3}$. Linear static analysis using ADINA has been carried out under self-weight condition.

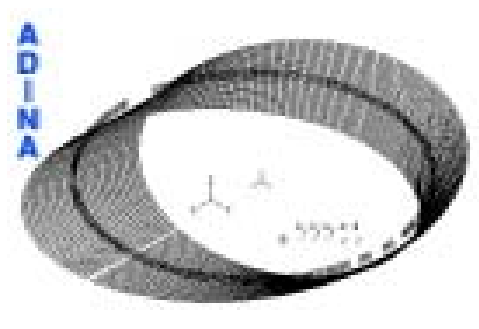

(a) Case I

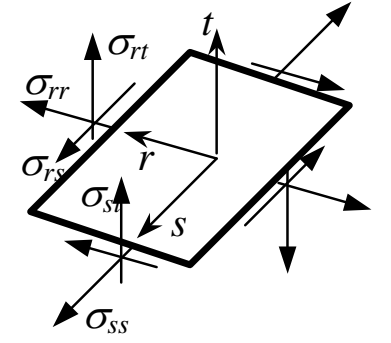

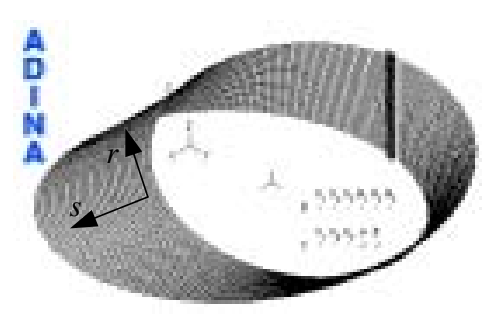

(b) Case II

Figure 6: FE models of Möbius strip shell surface used in numerical analysis

Distribution of stress and displacement are examined. Maximum values of stress and displacement in Case II are larger than those of Case I as can be seen in the tables of Figure 7. The notations of stress components are as shown in Figure 6. Note that axis $t$ is perpendicular to the surface.

\begin{tabular}{|c|c|c|c|}
\hline Case & $\begin{array}{c}\text { Max. } \mathrm{d}_{\mathrm{z}} \cdot \\
(\mathrm{mm})\end{array}$ & $\begin{array}{c}\text { Max. } \sigma_{\text {rr }} \\
(\mathrm{MPa})\end{array}$ & $\begin{array}{c}\text { Max. } \sigma_{\mathrm{ss}} \\
(\mathrm{MPa})\end{array}$ \\
\hline I & $1.9(0.0)$ & $0.64(0.64)$ & $1.95(1.95)$ \\
\hline II & $19.6(4.6)$ & $42.4(15.67)$ & $6.46(12.13)$ \\
\hline
\end{tabular}

(a)

\begin{tabular}{|c|c|c|c|}
\hline Case & $\begin{array}{c}\sigma_{\text {rs }} \\
(\mathrm{MPa})\end{array}$ & $\begin{array}{c}\sigma_{\text {rt }} \\
(\mathrm{MPa})\end{array}$ & $\begin{array}{c}\sigma_{\text {st }} \\
(\mathrm{MPa})\end{array}$ \\
\hline $\mathrm{I}$ & $0.255(-0.255)$ & $0.126(-0.0354)$ & $0.148(-0.091)$ \\
\hline $\mathrm{II}$ & $10.8(-4.39)$ & $6.50(-6.99)$ & $0.147(-3.50)$ \\
\hline
\end{tabular}

(b)

Figure 7: Maximum values of stress and displacement in Case I and Case II

Bracketed values in table of Figure 7 a denote upward displacement for maximum vertical displacement $\left(\mathrm{d}_{\mathrm{z}}\right)$ and tensile stress for membrane stresses $\sigma_{\mathrm{rr}}$ and $\sigma_{\mathrm{ss}}$.

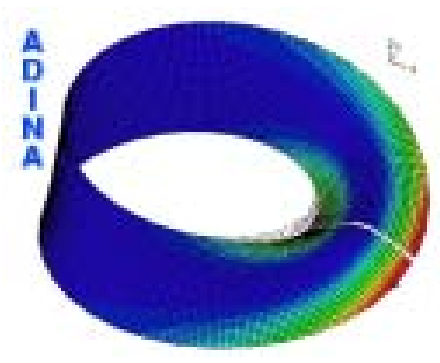

(a) Case I
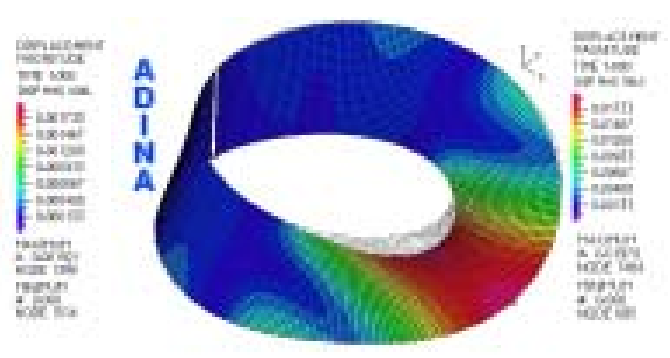

(b) Case II

Figure 8: Distribution of displacement magnitude : (a) Case I and (b) Case II

From Figure 8, it can be seen that maximum displacement occurs at portion of the shell surface where the inclination with respect to horizontal plane is small. Taking ratio with free-span of each case, i.e. (i) Case I: $1.9 / 3500 \approx 1: 1842$ and (ii) Case II: $19.6 / 7000 \approx 1: 357$, it can be concluded that the maximum vertical displacement in both cases is acceptable. 
Assuming grade of concrete at the lower end normally used for construction, e.g. concrete with characteristic strength of $\mathrm{f}_{\mathrm{cu}}=25 \mathrm{MPa}\left(\sigma_{\mathrm{C}, \text { all }}=25 / 1.5=16.7 \mathrm{MPa}, \tau_{\mathrm{all}}=0.8 \sqrt{ }\left(\mathrm{f}_{\mathrm{cu}}\right)=4 \mathrm{MPa}\right.$, where $\sigma_{\mathrm{C} \text {,all }}$ and $\tau_{\text {all }}$ are allowable compressive and shear stress, respectively), it can be seen from tables in Figure 7 that all maximum values of stress in Case I fall within the allowable limit except maximum tensile stress of $\sigma_{\mathrm{rr}}$ and $\sigma_{\mathrm{ss}}$. However, comparison with the corresponding maximum values of stress in Case II shows that allowable limit are exceeded for all cases except for $\sigma_{\mathrm{st}}$. Figures 9 and 10 show the distribution of in-plane stresses $\sigma_{\mathrm{rr}}, \sigma_{\mathrm{ss}}, \sigma_{\mathrm{rs}}$ and out-of-plane stress $\sigma_{\mathrm{rt}}$, respectively for Case II. From the two figures, it can be seen that extreme values of stress concentrate at region at and in the vicinity of support point. Such extreme values can be properly overcome by thickening of shell surface or introduction of distributed instead of concentrated support.

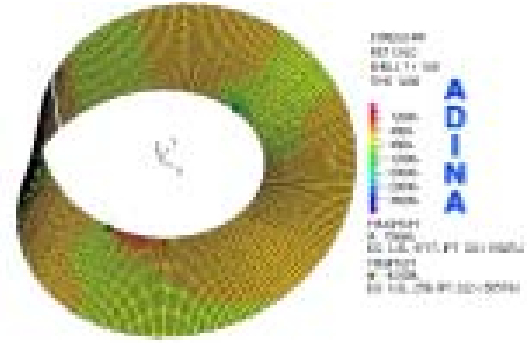

(a)

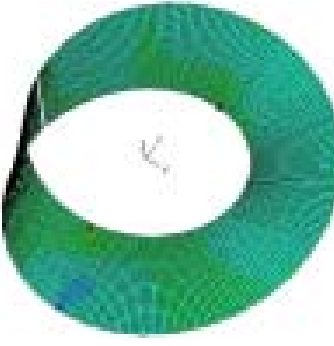

(b)

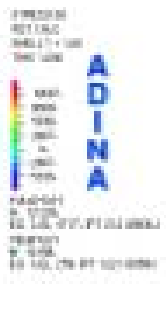

Figure 9: Distribution of in-plane stress in Case I : (a) $\sigma_{\mathrm{rr}}$, (b) $\sigma_{\mathrm{ss}}$ and (c) $\sigma_{\mathrm{rs}}$

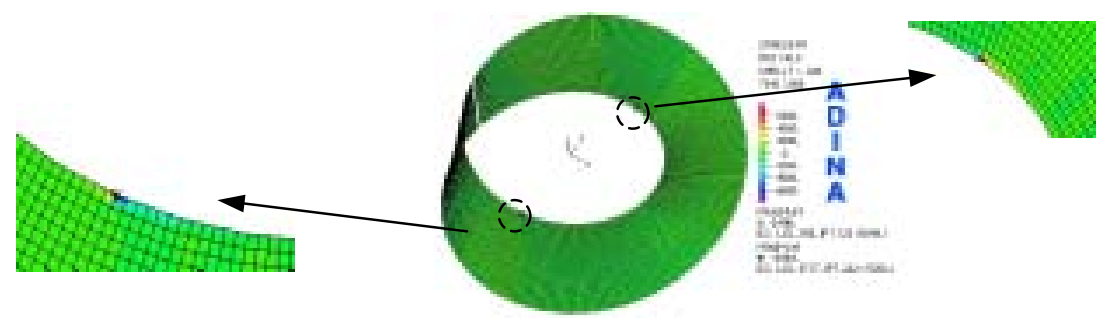

Figure 10: Distribution of out-of-plane stress $\sigma_{\mathrm{rt}}$

\section{Conclusion}

A computational study has been carried out on a shell surface in the form of Möbius strip. Numerical results show that support condition plays a significant role on the strength and stiffness of the surface. Furtherwork in identifying optimum support condition from the view point of structural performance is needed.

\section{Acknowledgement}

The supports by Universiti Sains Malaysia in the form of a short term research grant and Professor Kawaguchi Ken'ichi of The University of Tokyo in providing facility for using ADINA software is highly appreciated.

\section{References}

[1] Friedman, Nathaniel A. Hyperseeing, Hypersculptures, Knots and Minimal Surfaces. http://members.tripod.com/vismath7/nat/index.html (accessed March 14 2008)

[2] Peterson, Ivars. Möbius in the playground. http://www.maa.org/mathland/mathtrek_5_24_99.html (accessed March 14 2008)

[3] Séquin, Carlo H. To Build a Twisted Bridge. http://www.mi.sanu.ac.yu/vismath/sequin/index.html (accessed March 14 2008)

[4] Weisstein, Eric W. Möbius Strip. From MathWorld--A Wolfram Web Resource. http://mathworld.wolfram.com/MoebiusStrip.html (accessed March 14 2008) 


\title{
On the interaction between architecture and engineering: the acoustic optimization of a reinforced concrete shell
}

\author{
Mario SASSONE*, Tomàs MENDEZ, Alberto PUGNALE, \\ *Assistant Professor, Department of Structural and Geotechnical Engineering, Politecnico di Torino \\ 10125 - Viale Mattioli 39, Torino, Italy \\ mario.sassone@polito.it
}

\begin{abstract}
In this paper a computational morphogenesis process is applied to the design and the optimization of the shell roof of a large music hall. The architectural concept is based on the folded plate technique: a set of reinforced concrete plates, connected to each other on the edges, forms the global shape of the roof. The dimensions of each plate are different, so that the structure is not regular; moreover each plate is not plane but slightly curved to form hypars. Starting from the geometric concept of the roof, a process of optimization and form finding has been applied, in order to obtain the best acoustic performance, in terms of distribution of the acoustic pressure level in the hall. The folded plate surface has been described mathematically by using a NURBS representation and the control point positions has been assumed as the design variables. The acoustic performance has been evaluated by means of an algorithm able to calculate the acoustic pressure level in all the points of the hall, considering the reflection of the roof. The uniformity of acoustic pressure level in all the points of the hall has been assumed as the target of the optimization process. A genetic algorithm, i.e. an evolutionary iterative population-based scheme, has been adopted in the optimization procedure. The evolution of the shape towards the optimal solution is controlled interactively by the designer, in order to avoid the convergence to unexpected configurations.
\end{abstract}

\section{Introduction}

Architectural design is a complex multidisciplinary activity, involving many different professional competencies and actors. Due to the development of digital technologies, the competencies frequently express themselves in terms of mathematical models of problems and of sophisticated computational tools able to give the solutions (Migayrou et al. [5], Sassone [9]). Structural engineering is perhaps one of the disciplines where the numerical tools, as the FEM analysis has had this large diffusion, but nowadays computational applications are available for a wide range of problems, from photorealistic rendering to design organization, from lighting control to hygrothermal design, from structures to acoustics. As studies on that topic show, (Frazer [2]), the interaction between different problems is then mediate by a continuous exchange of information, representing a coded description of the problem. Such interaction can be intended as an extension of the multiphysics approach form the study of the physical world, to the design of the human environment (Michopoulosa et al. [4]). In this paper the multidisciplinary approach has been applied to a problem of acoustic design, by coupling the technology of acoustic analyzers to an optimization strategy based on genetic algorithms.

\section{The analysis of acoustic behavior}

Besides the earlier empirical methods, as the Sabine prediction of time reverberation, two main computational approaches are generally adopted in the analysis of the response of an acoustic enclosure. The first one is based on the approximate solution of the wave equations over the space domain represented by the enclosure. Finite Elements Method, Boundary Elements Method or Finite Differences Time Domain Method are the most common numerical schemes of solution. A second approach, introduced by Kronstad et al. [3], is based on the geometrical description of sound propagation paths; reflection, absorption and diffusion are then modeled as 
modifications of the sound energy travelling along paths and the procedure is perfectly analogue to the raytracing technique in photorealistic rendering. This second approach is much more efficient in terms of computational effort with respect to wave equations solution, but a set of phenomena can not be correctly represented. One of them is interference, that only appears if sound is described in terms of waves.

In order to apply an evolutionary optimization technique to the acoustic analysis, the problem of interfacing two different computational tools has been solved by developing a customized ray-tracing acoustic algorithm inside the $3 \mathrm{D}$ geometrical modeler. This has been possible thanks to the nature of the acoustic problem, that is, when modeled following the geometrical approach, intrinsically analogue to a lighting analysis problem. The sound propagation from the source to the receiver is geometrically described as a path made of straight line segments, which is generated following the reflection rule with an assumed ratio of absorption. The analysis is of course simplified, but the algorithm is able to guide the optimization process to better solutions, that can be lately checked with more sophisticated acoustic analyzers.

\subsection{The Ray-Tracing procedure}

In its basic configuration a Ray-Tracing algorithm is composed by a source, a receiver and a set of reflecting objects, representing the environment. Each ray starts from the source with a defined or random direction and travels until it meet the first reflecting object. Here is reflected and restarts with an angle equal to the incidence angle, until it reaches a new reflecting object or the receiver.

The receiver is generally a surface on which rays arrive with a distribution depending on the way they are distributed in the source and on the characteristics of the reflecting (and absorbing) environment. The source can be directional, it means that the density of sound energy is higher in one direction that in the others, or perfectly uniform. Because each ray is assumed to carry the same amount of energy, in a uniform source each direction has the same probability. The energy, on the other hand, is reduced by absorption every time the ray is reflected by a surface and the energy level on the receiver depends on the adsorption too.

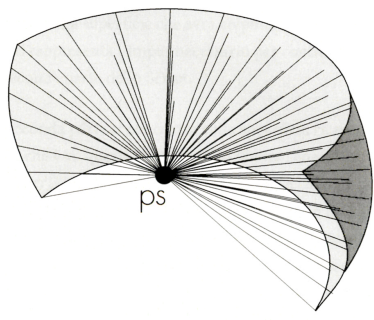

Figure 1: Spherical sector for the ray-tracing computation;
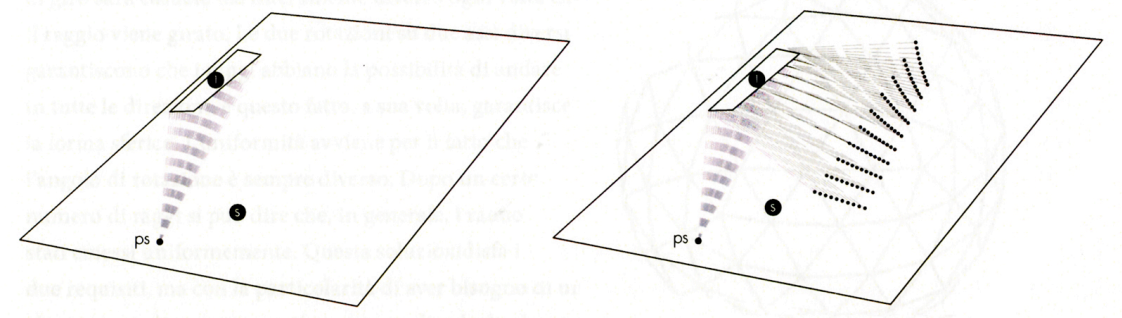

Ray-tracing method from a single point source.

\subsection{Evaluation of the acoustic performance}

From the distribution of the rays on the receiver it is possible to obtain the distribution of the energy density. If the receiving surface corresponds to the public seats area of the hall, a good acoustic performance can be related to the uniformity of the sound level in this area, necessary to provide a good listening position to all the listeners.

In order to evaluate uniformity, the area is divided into sections and the sound energy corresponding to each section is calculated by summing the contribution of each ray falling in the section. This contribution can be less or equal to one, assuming that one is the contribution without any adsorption or attenuation. Generally each section represent one listener, therefore their dimension should be of about 50x50 centimeters (the average dimension of an audience seat).

The uniformity of sound distribution in the listeners area is then evaluated by comparing the amount of energy reaching each listener, assuming that the sound distribution will be uniform if every listener receives the same amount of sonic energy. The inverse of standard deviation of sonic energy values is then assumed as an indicator of the acoustic performance. If the optimal distribution is not uniform, i.e. if a specified non uniform distribution is desired, the differences between the desired an the actual values of energy have to be considered. 
The acoustic performance, intended as a measure of the difference between the actual surface acoustic response and the optimal one becomes the parameter on which the evolutionary form finding algorithm is based.

At the stage of the optimization process, the use of a simplified acoustic analyzer, instead of a more complex commercial application, allows a better control of the optimization process and the designed can easily interact with the process itself. A detailed acoustic analysis of the building, at the final design stage, should be performed with the aid of a specialized digital tool by the acoustic consultant.

\section{The genetic algorithm}

The structure of the evolutionary algorithm used in the optimization process is described in more detail in Pugnale and Sassone [7][10]. The adopted Genetic Algorithm assumes the design variables encoded as real numbers, the classical roulette wheel operator for the selection of best individuals and a two dimensional crossover and random mutations as operators to search new more efficient solutions.

It is to underline that the same Genetic Algorithm can be used for different optimization problems only implementing the function for the specific performance evaluation inside the general code. Indeed, the design variables and the geometrical description of the shapes is read by the algorithm from a matrix of coordinates of the surface control points.

\section{Application to a case study}

The case study is a 900 seat concert hall designed for an architectural senior thesis. Figure 2 shows the base surface for this concert hall. It is defined by 24 geometric points. These points define the 15 elements of the surface. The variables in play will be the $\mathrm{X}, \mathrm{Y}$ and $\mathrm{Z}$ coordinates of each one of the 24 points. This means that each point will be free to move in three dimensions during the form finding process, although movement will be restricted by user defined maximum and minimum distances in order to avoid points overlapping and in also to contain the surface within the space limitations of the hypothetic concert hall. Each surface is described, in order to apply the acoustic analyzer, as a NURBS continuous surface (Piegl et al. [6]) son that the analysis of reflections can be improved just by increasing the number of sound rays, obtaining a very high resolution.

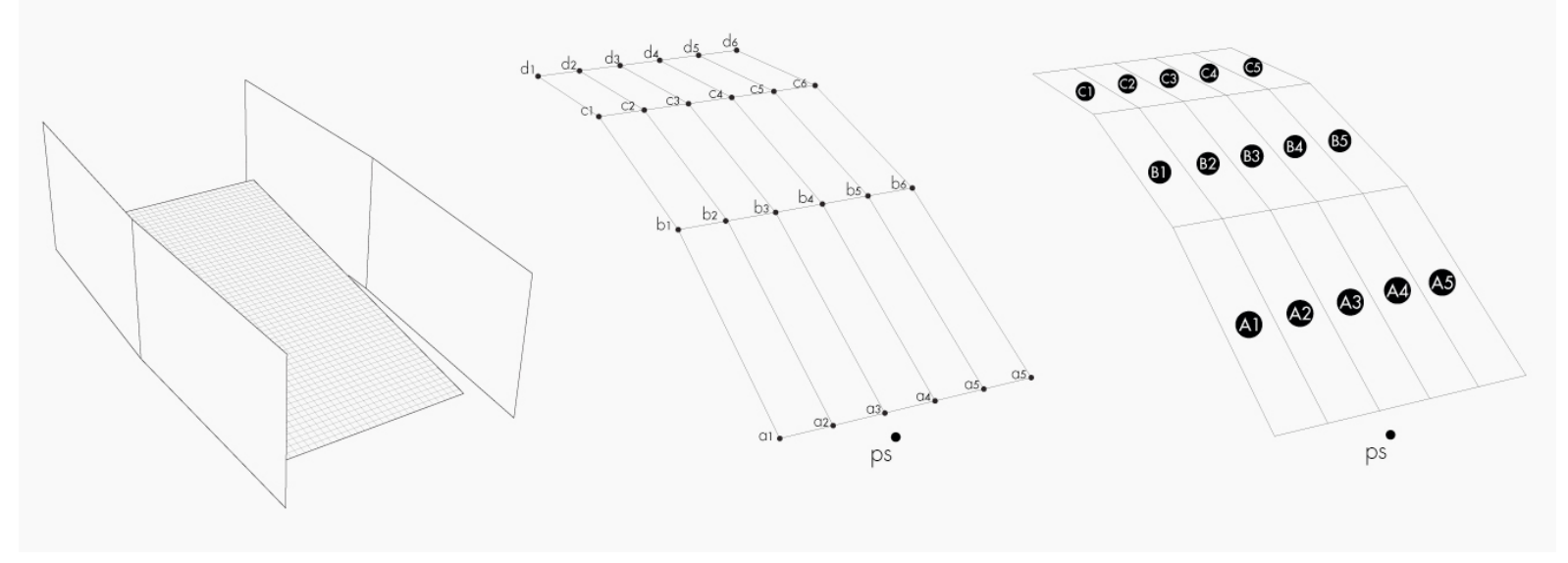

Figure 2: The seats area and lateral walls; Control points of the roof surface; Elements of the roof surface.

The evolutionary procedure starts by defining the audience area and the concert hall walls. Then it calculates the amount of energy that each audience member receives directly from the source and reflected by the hall walls, on the basis of the listener's position. The energy is calculated only once for all of the surfaces forming the population in acoustic simulations, because these values do not change with the different surfaces. The analyzer then calculates the acoustic fitness values for the different surfaces. Besides the 15 elements roof, a set of analyses and optimization cycles have been performed on simpler cases, in order to better understand what factors affect the acoustic performance. In figure 3 the distribution of rays on the receiver is shown for a four elements roof, at one step of the optimization procedure ordered by increasing uniformity from left to right. 

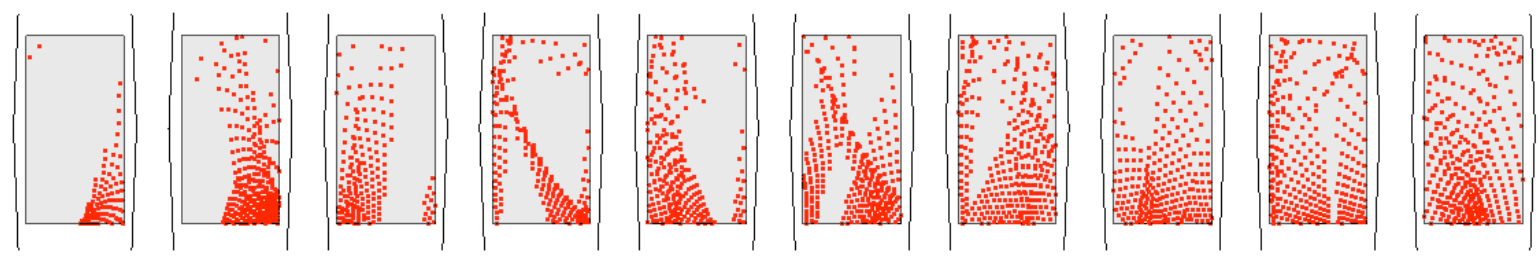

Figure 3: Acoustic response of the individuals of one generation of roof shapes.

The early result of the optimization procedure, show that the algorithm, depending on how the solution domain is wide or narrow, is able to generate a large set of optimal or sub-optimal solutions, i.e. solutions in which the acoustic distribution is close enough to uniformity, with different shapes, less or more regular, less or more responding to the architectural and formal requirements. In fact, when the architectural concept contains a defined amount of uncertainty and randomness, population based optimization algorithms can take advantage from the "freedom" included in the concept, to produce efficient solutions. In this sense evolutionary optimization is not limiting the architectural concept, but interact actively with it.

\section{Future developments of the research}

The main advantage in using a Genetic Algorithm developed inside a three dimensional modeler is the possibility for the designer to keep an high interaction with the optimization process at each step of the procedure. This kind of interaction is possible even with other evolutionary algorithm (Elbeltagi et al. [1], Renner et al. [8]), and the research is developing in the application of different computational strategies, in order to improve the efficiency and the robustness of the algorithm.

A second development is related to coupling the acoustic and structural optimization, by applying the approach already shown in Pugnale and Sassone [7], by applying a multi-objective optimization approach.

\section{References}

[1] Elbeltagi E, Hegazy T, Grierson D. Comparison among five evolutionary-based optimization algorithms, in Advanced Engineering Informatics 2005; 19: 43-53.

[2] Frazer JH. An Evolutionary Architecture, Architectural Publications Association, 1995.

[3] Krokstad A, Strom S, Sorsdal S. Calculation of the acoustical room response by the use of a ray tracing technique. Journal of Sound and Vibrations 1968, 8: 118-125.

[4] Michopoulosa J, Tsompanopouloub P, Houstisb E, Farhatd C, Lesoinned M, Ricec J, Joshie A. On a datadriven environment for multiphysics applications, Future Generation Computer Systems 2005, 21: 953968.

[5] Migayrou F. Architectures non standard, Centre Pompidou, 2004.

[6] Piegl L, Tiller W. The NURBS book, 2nd edition, Springer, 1997 (1966).

[7] Pugnale A, Sassone M. Morphogenesis And Structural Optimization of Shell Structures with the aid of a Genetic Algorithm. Journal of the International Association for Shell and Spatial Structures 2007; 48: 161-166.

[8] Renner G, Ekàrt A. Genetic Algorithms in Computer Aided Design. Computer Aided Design 2003, 35: 709-726.

[9] Sassone M. Computational Morphogenesis in Grid Shell Design. In Accommodating new Aspects of Interdisciplinarity, Voyatzaki M. (eds.) EAAE Transactions: Thessaloniki, 2006; 479-485.

[10] Sassone M, Pugnale A. Optimal design of glass grid shells with quadrilateral elements by means of a genetic algorithm. In Proceedings of the 6th International Conference on Computation of Shell and Spatial Structures IASS-IACM 2008, Abel JF, Cooke JR (eds.): Ithaca NY, 2008. 
Proceedings of the 6th International Conference on Computation of Shell and Spatial Structures

IASS-IACM 2008: "Spanning Nano to Mega"

28-31 May 2008, Cornell University, Ithaca, NY, USA

John F. ABEL and J. Robert COOKE (eds.)

\title{
Singularities
}

\author{
Peter MACAPIA* $^{*}$, Frank BITONTI, Robert BAKER, Charles KWAN \\ *Pratt Institute GAUD/ Columbia University GSAPP
}

136 Hicks Street no. 4G, Brooklyn NY 11201, USA

petermacapia@labdora.com

\begin{abstract}
This paper describes the way in which an architect uses engineering software and algorithms within modeling design programs to research problems of structural organization. The advent of computational techniques, from genetic algorithms to random search spaces, enables us to consider variations that might be overlooked within conventional engineering approaches to structural design. Specifically, we have looked at the way in which orthogonal grids might be reconsidered from a more fluid approach to structural thinking by introducing gradients and singularities. The process involves comparing orthogonal control grids with distorted grids using FEA and the use of stress differences to vectorize zones. These zones are then imported to a new program for which the authors have developed an algorithm script that associates in the form of a continuous function, points on a grid to move according to the zones of stress. The new grid is then re-analyzed again with FEA, this time a series of algorithms are introduced to identify different types of singularities within the system. In basic terms, the computational system incorporates a gradient function to optimize a structural grid in order to develop within it singularities that can then function as beams and columns. The recent development of this research, however, expands on the concept of singularities to include first, second, and third order singularities. These different types of singularities correspond to features of density, member thickness, and topological continuity. Our goal is to further expand this research to include other "species" of singularities that optimize structural organization. Keywords: Singularity, Topology, Structure, Architecture, Algorithm
\end{abstract}

\section{Introduction}

The following studies describe a series of experiments using Finite Element Analysis and different forms of script writing (computer programming) for the development of structural meshes. Our initial experiments (Fig. 1 and 2) involve developing an algorithm for selecting, transforming, and retesting the grid structures. Part of this research project has involved developing custom software that uses agent based self-organizing systems to search out intensive singularities within the non-metric search space of structural loads and resulting forces. The software couples powerful $3 \mathrm{~d}$ modeling software with engineering grade analysis tools.

The software first translates the $3 \mathrm{~d}$ geometry into a data set that can be easily imported into an analysis application. After calculations are performed, geometric information (the topological connectivity of nodes) is paired with the resulting stresses. A matrix of data is created and shared between applications. Our software uses principles similar to those employed in two dimensional cellular automata to navigate a search space of structural and geometric data.

Singularities in these intensive search spaces are defined through patterns of behavior. As automatons operate within and respond to an environment of forces. The software can begin to see particular patterns emerge within the two dimension matrix described above. For example, a certain number of tension members clustered around a member in compression, or a specific rate of change in the stresses between neighboring members.

Upon locating a singularity within the dataset, geometric transformations, or rather geometric singularities can be automatically manifested within the $3 \mathrm{~d}$ modeling application. Depending on what the designers' objective is in deploying the geometric singularity, intensive singularities will need to constantly be redefined. Essentially 
the software operates as an intermediary merging the functionality of two different applications. In the service of helping the designer redefine their relationship between analytical and geometric applications and thereby fostering new attitudes about geometric and material organization.
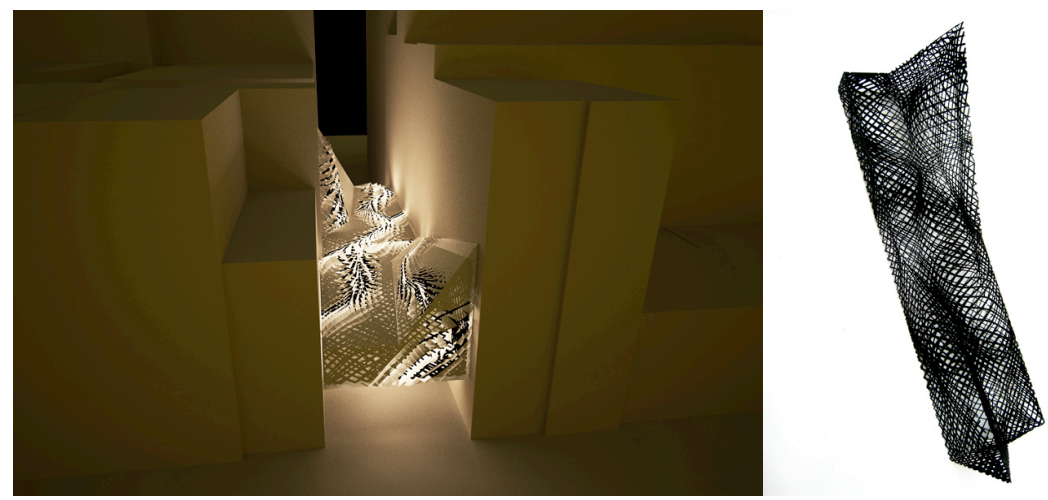

Figure 1: Rendering and study model of First and Second-order Singularity grid.

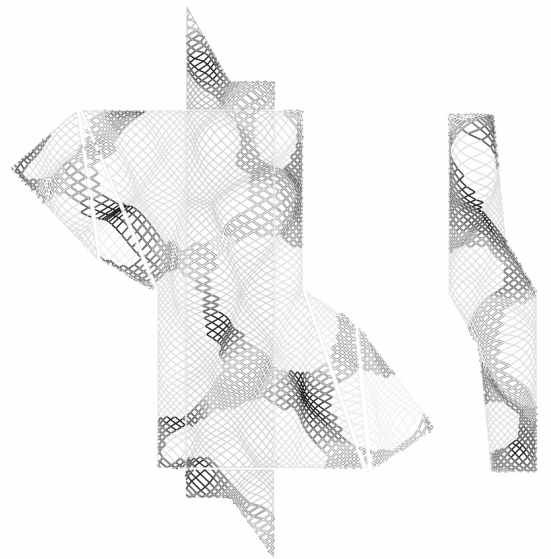

Figure 2: CAD drawing of First and Second-order Singularity members and their topological organization.

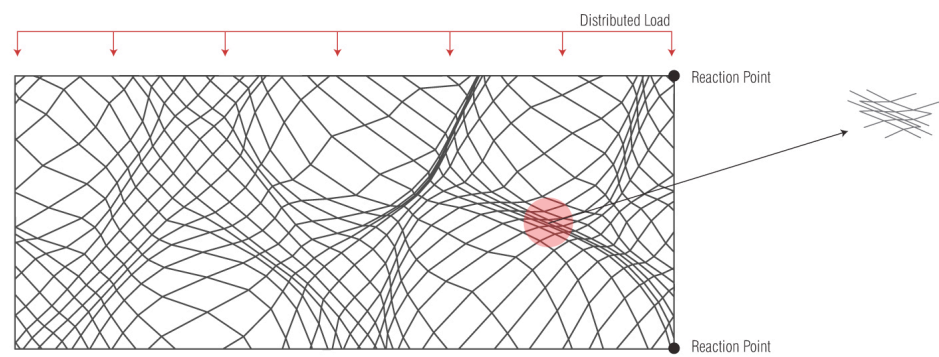

Figure 3: FEM study of First-order Singularity system.

In the first order singularity system (Fig. 3) members are transformed through the analysis of local conditions member by member. Member length and position of each member is then adjusted based on simple rules set to produce a gradient structure of densities. The cantilever is a homogeneous orthogonal mesh which is then tested and the member displaced to optimize the cantilever. Notice how the new mesh resembles a regular $2 \mathrm{D}$ truss. 

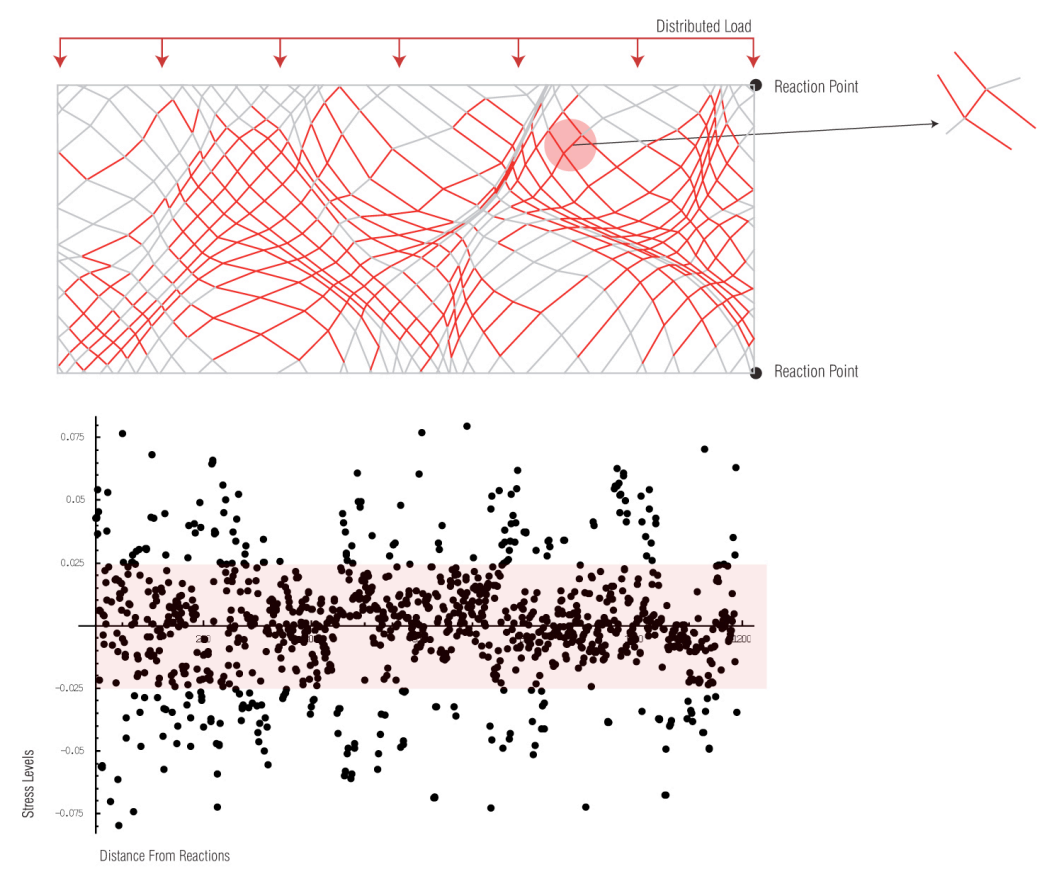

Figure 4: Second order singularity system.

In the second-order singularity system (Fig. 4) members are selected as a function of a range of stress values and the member size adjusted. The software iterates through the mesh and evaluates the stress value for each member and then calculates the difference between that member and each of it's neighbors. Transformations are applied if the difference between a member and it's neighbor reach a specific value. Rate $=$ Member 1 Member 2. Each of those values are on the graphs bellow as the program spiders it's way through the mesh.
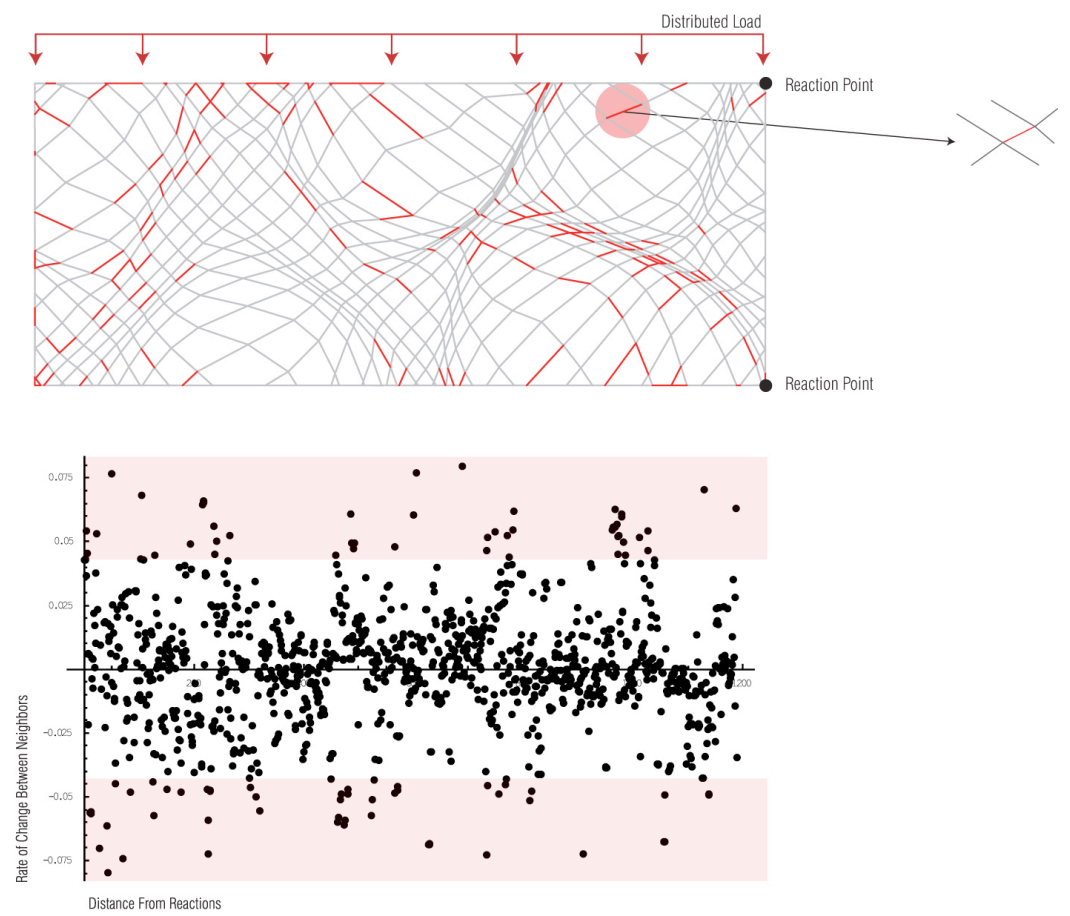

Figure 5: Third Order Singularity System 
In the experiment with the Third-Order Singularity system rather then strictly defining a target rate of change the software defines the target rate of change from the current local condition for example the user sets a target difference. As the stress of each member changes the target will constantly fluctuate based on local conditions. The member is selected only if it falls within the specified range between the average of all six of it's neighbors. Essentially the program will find the areas of the mesh where the stress levels fluctuate the least regardless of the specific stress level of each member.
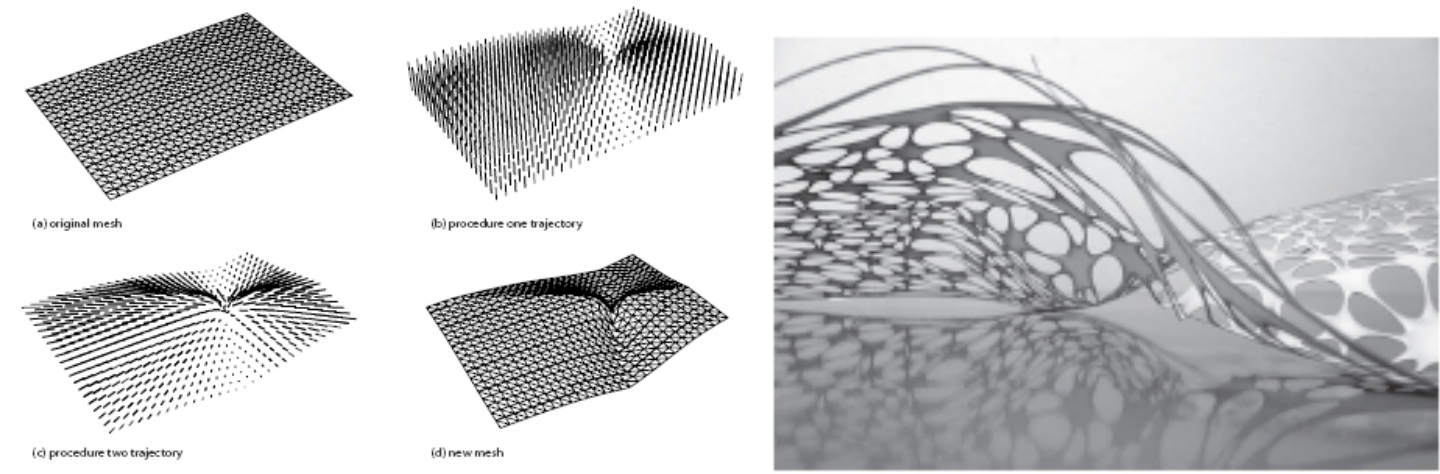

Figure 6: Out-of-plane Singularity System

The last set of experiments (Fig 6) look to establish a structural hierarch with singularities that move out of plane. This script was developed with the goal of generating a pinching condition of the mesh. The inputs are the mesh, and the variables are pinch point on mesh, target, and the intensity of the deformation. The interest of this research began with the material studies of a 2-dimensional mesh that is physically pulled at different points to generate deformations. This deformation of the mesh allows it to go from a planar condition to a spatial condition. Beginning with the cutting of one member of a flat physical mesh and relocating the exposed end to a new position in space by joining it to the node of the neighboring cell, the designer observes the redistribution of every node throughout the entire mesh in space relative to the localized transformation. searching for an underlying geometric pattern to describe this change, the designer finds that the overall material behavior of the mesh can be approximated digitally through the iterative application of two procedures for each node: first, the position of each node is to be added to a vector normal to the plane of the transformation. secondly, this new point is added to a vector oriented towards the center of the transformation with a length proportional to the distance from the cut and glue points of the transformation. to account for the observed variation in the degree of relocation for each node of the mesh, the vectors used in these procedures are scaled by a factor proportional to the angle between a line from their current locations and the center of the transformation and the line between the cut and glue points of the transformation. 
Proceedings of the 6th International Conference on

Computation of Shell and Spatial Structures

IASS-IACM 2008: "Spanning Nano to Mega"

28-31 May 2008, Cornell University, Ithaca, NY, USA

John F. ABEL and J. Robert COOKE (eds.)

\title{
Optimal structural shapes for shells using hybrid GA
}

\author{
Nidur SINGH, C. V. RAMAKRISHNAN*, D. K. SEHGAL \\ * Applied Mechanics Department, IIT Delhi Hauz Khas New Delhi, Delhi-16 \\ Email: cvrama@am.iitd.ernet.in
}

\begin{abstract}
The topology optimization problems for plates and shells are solved using Hybrid Genetic algorithm. The problem is formulated as volume minimization problem with several constraints on displacements. Results are compared with the artificial density method in terms of optimal shapes and computing effort.
\end{abstract}

\section{Introduction}

Optimal shapes for structure under mechanical loads are usually obtained first arriving at an optimal topology over a base mesh followed by a shape optimization for obtaining more precise and smooth shapes. Topology Optimization is usually carried out by one of the following method i.e, Homogenization method, Artificial Density method or GA. Homogenization and Solid Isotropic material with penalization (SIMP) approaches are widely used and some general purpose codes are available which use these methods. These methods usually consider stiffness maximization as the objective function with a volume constraint. Multiple loading condition problem is tackled by using a weighted objective function of different loads for arriving at the practical solutions quickly. With the availability of high performance computing clusters at affordable cost and improvements in GA involving the capturing of problem related intelligence and Two stage algorithm with adaptivity, it has become possible to target global optima.

Most of the authors have shown the generality of these methods using mostly 2D plane stress problems. Practical problems related to plates and shells have received limited attention $[1,2]$. The current work looks into topology optimization of such structures using efficient Hybrid GA. The results are compared with artificial density based solutions in terms of final shapes and computational cost.

\section{Description of Methods}

The design domain is discretized into a fixed number of finite elements of voids and solids and is represented through a binary string that is manipulated through the Genetic Algorithm-based optimizer for the optimum topology design. It is known that the GA generally converges very fast in the initial stages but further progress towards the global optimal solution is very slow. Balamurugan $[3,4]$ proposed the Two phase algorithm for topology optimization in which the first phase uses the more general bit-array representation and the second phase uses a geometric representation which is extracted on the basis of the converged skeleton of the previous phase.

As a further improvement, a three phase Hybrid GA proposed by the author [5] has been used in this investigation. The first phase is carried out using the widely used bit-array representation method that helps in searching for global optimal skeletons/topological connections without any restriction on the design space. This is carried out until the numbers of best topologies in last 100 generation are no more than a few. The topology which has the maximum number of nodes and branches is chosen as the base topology and geometric variables for these are extracted and it is ensured that all the best topologies constitute a subset of the base topology. Using these new variables the second phase of GA based optimization is resumed. Thus the description of design is transformed from large sized bit array to a small number of geometric variables. The selected general skeleton is considered as the starting point and geometric parameters of the selected skeleton are extracted and optimized. During this second phase the rectangular blocks representing the skeleton are allowed to move along a horizontal raster line. This movement of rectangles allows merging and opening of holes thereby allowing new topologies to emerge. This phase is continued until the skeleton convergence takes place, which can be 
identified through the automated convergence detection procedure. Once this is achieved the converged skeleton is used as the fixed topology for the third phase and the geometric variables of the skeleton are extracted and optimized in the third phase using GA. To accelerate convergence, this Third phase can be carried out using Improved Sequential Integer programming. All structural analyses were performed using FEM with nine noded Heterosis shell element.

The conventional approach called Solid Isotropic Material with Penalization uses power law variation of density for the characterization of material stiffness. To ensure existence of solutions, this approach must be combined with a perimeter constraint or a gradient constraint or with a filtering technique. The Sequential linear programming with move limits is used as the optimizer which has the advantage of incorporating multiple constraints in the problem. It is observed such a solution obtained has a high proportion of grey areas. These perforated (grey) areas have intermediate densities between 0 and 1 . These intermediate densities can be suppressed by penalizing them appropriately by adding a barrier function to the objective function. Problems with Multiple loading conditions have been solved with the objective function being weight subject to constraints on displacements as described in section 3. The problem is solved in two stages. In first stage the problem is run without the penalty function. The algorithm smoothly converges to a void-fill design if the starting design in the second phase is not too close to any local optimum. Design Derivatives computations of constraints with respect to design variables is done using adjoint method. Two approaches were tested, one in which optimization mesh and Finite Element mesh were the same and in other four optimization elements were used in one finite element for analysis. The sensitivities obtained by using the adjoint method in both the cases were thoroughly checked for accuracy using the finite difference method.

\section{Mathematical formulation of the Structural Topology Optimization Problem}

\subsection{Topology Optimization formulation using GA}

The topology optimization problem can be stated as,

$\min V=\sum_{e=1}^{N} \chi_{e}(x) V_{e}$

Subject to

$$
g_{j}=\frac{a_{p_{j}}}{a_{p_{j a}}}-1<0 \quad \mathrm{j}=1 \text { to } \mathrm{m} \text { and } \frac{P-P_{a}}{n_{2}} \leq 0
$$

And $K \bar{a}=\bar{f}$

$V_{s}, P$ are solid volume, total perimeter of a design respectively and $V_{a}, P_{a}$, are the corresponding allowable values. $\bar{f}$ denotes the nodal load vector and $\bar{a}$ is the vector of global nodal displacements in the FE formulation. $a_{p_{j}}$ and $a_{p_{j a}}$ are the $p_{j}^{\text {th }}$ displacement component and its limiting value. $\chi_{e}$ and $V_{e}$ are the indicator function value (zero or one) and the volume associated with discrete element ' $e$ '. Alternatively, if only a single compliance constraint is considered, the dual formulation can be used which can be solved using optimality criteria based iterative technique.

\subsection{Topology Optimization problem using SIMP}

In topology optimization problem based on the power law approach (SIMP), the material stiffness is assumed to obey power law

$$
\begin{array}{ll}
K_{e}=\rho_{e}^{p} K_{0} & \text { and } \quad K=\sum_{e=1}^{n} K_{e} \\
V_{e}=\rho_{e} A_{0} & V=\sum_{e=1}^{n} \rho_{e} A_{0} \quad \text { and } \quad 0<x_{\min } \leq \rho_{e} \leq x_{\max } \leq 1
\end{array}
$$

The Objective function and constraints are the same except that $\rho_{\mathrm{e}}$ replaces $\chi_{e}$ are easily derived and computed. Here $\rho_{\mathrm{e}}$ is the vector of design variables, $\mathrm{x}_{\min }$ and $\mathrm{x}_{\max }$ is a vector of minimum and maximum relative densities (non-zero to avoid singularity), $\mathrm{N}$ (= nelx $\mathrm{X}$ nely) is the number of elements used to discretize the design domain, $\mathrm{p}$ is the penalization exponent (typically $\mathrm{p}=5$ ), $\mathrm{V}_{\mathrm{s}}$ and $\mathrm{V}_{0}$ is the material volume and design domain 
volume, respectively and $\mathrm{V}_{\mathrm{f}}$ (volfrac) is the prescribed volume fraction $\left(\mathrm{V}_{\mathrm{s}} / \mathrm{V}_{\mathrm{o}}\right)$. The derivatives of the objective function constraints can be derived as follows.

\subsubsection{Design Derivative Calculation}

\subsubsection{One Design varaible in one FE Element}

The Design derivatives have been calculated using the Adjoint method.

$$
\begin{aligned}
& \frac{d g_{j}}{d \rho_{i}}=\lambda^{T} \frac{\partial R}{\partial \rho_{i}} \text { where } R=\bar{f}-[K] \overline{a_{i}} \\
& \frac{\partial R}{\partial \rho_{i}}=-\frac{p}{\rho_{i}}[K]_{i} \bar{a}_{i} \text { where }[K]_{\mathrm{i}} \text { and } \bar{a}_{i} \text { are the stiffness matrix and the element displacement vector of }
\end{aligned}
$$

element ' $\mathrm{i}$ '. $\lambda^{T}$ is the vector of Lagrangian multipliers which can be obtained by solving $K \lambda=P$ where $P$ is unit load vector applied at the constrained node.

\subsubsection{Four Design variable in one FE Element}

Here four material variables $\rho_{\mathrm{i}}(\mathrm{i}=1,2,3,4)$ have been used for each nine-noded Heterosis shell element. $\mathrm{D}_{\mathrm{o}}$ is the elasticity matrix

$\frac{\partial R}{\partial \rho_{i}}=-\frac{p}{\rho_{i}} \sum_{i g=1}^{9}\left[B_{i g}^{T} D_{o} B_{i g}\right] \rho_{i g}^{p} \alpha_{i g} \overline{a_{i}}$

Figure 1 shows a FE element in which there are four density design variables $\rho_{1}, \rho_{2}, \rho_{3}$ and $\rho_{4}$. Each FE element has nine gauss points ig $=1$ to 9 . Table 1 shows the gauss points yielding non-zero contributions for each design derivative. The $\rho_{i g}$ and $\alpha_{i g}$ to be used at each gauss point is shown in Table 2.

\begin{tabular}{|l|l|l|l|l|}
\hline Residue Derivative & $\frac{\partial R}{\partial \rho_{1}}$ & $\frac{\partial R}{\partial \rho_{2}}$ & $\frac{\partial R}{\partial \rho_{3}}$ & $\frac{\partial R}{\partial \rho_{4}}$ \\
\hline Contributing Gauss Points & $1,2,4,5$ & $4,5,7,8$ & $2,3,5,6$ & $5,6,8,9$ \\
\hline
\end{tabular}

Table 1: Design derivatives and gauss points with non zero contributions

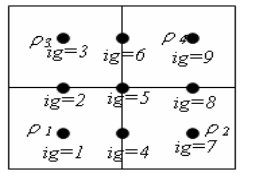

Figure 1: FE Element

\begin{tabular}{|c|c|c|c|c|c|c|c|c|c|}
\hline Gauss points & 1 & 2 & 3 & 4 & 5 & 6 & 7 & 8 & 9 \\
\hline$\rho_{\text {ig }}$ & $\rho_{1}$ & $\frac{\rho_{1}+\rho_{3}}{2}$ & $\rho_{3}$ & $\frac{\rho_{1}+\rho_{3}}{2}$ & $\frac{\rho_{1}+\rho_{2}+\rho_{3}+\rho_{4}}{4}$ & $\frac{\rho_{3}+\rho_{4}}{2}$ & $\rho_{2}$ & $\frac{\rho_{2}+\rho_{4}}{2}$ & $\rho_{4}$ \\
\hline$\alpha_{\text {ig }}$ & 1 & 0.5 & 1 & 0.5 & 0.25 & 0.5 & 1 & 0.5 & 1 \\
\hline
\end{tabular}

Table 2. Multiplication factors $\alpha_{i g}$ and equivalent $\rho_{i q}$ at particular gauss point

\section{Results and comparison}

\subsection{Weight Minimization problems}

A spherical shell as shown in figure 2. It is subjected to a concentrated laod at the centre and four at the quarter points of the lines of symmetry.For solving the topology optimization only one quarter of the problem is considered for optimization. The spherical shell is derived from sphere of radius of $20 \mathrm{inch}$ and side is 5inch and thickness of 0.05 inch. Displacement constraints are applied at point of loads application i.e, 1,2 and 3. Two problems are solved with different displacement constraints. Table 3 gives the details of the problem. The Optimum result of problem 4.1.1 is shown in figure 3. Figure 3(a) shows the opotimum design obtained by GA and Figure 3(b) shows the opotimum design obtained by SIMP method.

\begin{tabular}{|l|c|c|c|c|c|c|}
\hline \multirow{2}{*}{ Problems } & \multicolumn{3}{|c|}{ Loads (lb) } & \multicolumn{3}{c|}{ Displacements Constraints (inch) } \\
\cline { 2 - 7 } & At 1 & At 2 & At 3 & At 1 & At 2 & At 3 \\
\hline Problem 4.1.1 & 0.1 & 0.1 & 0.1 & 0.5 & 0.7 & 0.7 \\
\hline Problem 4.1.2 & 0.1 & 0.1 & 0.1 & 0.5 & 1.0 & 1.0 \\
\hline
\end{tabular}

Table 3. Problem definition of Spherical shell problems 
The Optimum result of problem 4.1.2 is shown in figure 4. Figure 4(a) shows the opotimum design obtained by GA and Figure 4(b) shows the opotimum design obtained by SIMP method. The results are tabulated in table 4.

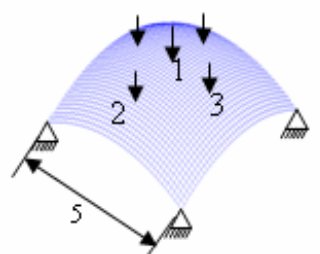

Figure 2: Spherical shell Problem

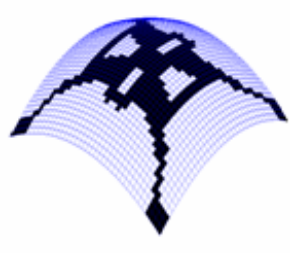

Figure 3(a) GA result $\left(\mathrm{V}_{\min }=0.3\right)$

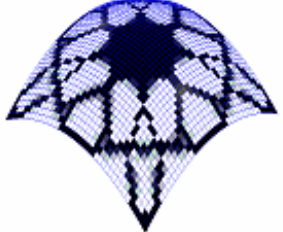

Figure 3(b) SIMP result $\left(\mathrm{V}_{\min }=0.4275\right)$

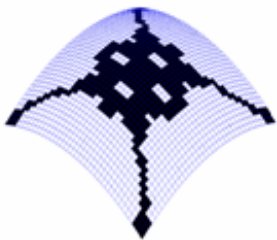

Figure 4(a)

GA result

$\left(\mathrm{V}_{\min }=0.2875\right)$

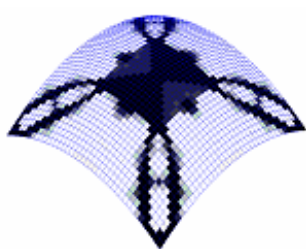

Figure 4(b)

SIMP result

$\left(\mathrm{V}_{\min }=0.3525\right)$

4.2 Comparison of Result of Three Phase Hybrid GA with SIMP

Table 4 shows the comparison of results obtained for both type of problems. It is be observed that SIMP methods has clearly scored over the problems stiffness maximization subject to volume constraints but $G$ A methods has performed well for problem Weight minimization problems. The reason of SIMP methods performing good in stiffness maximization problem is that they are single constraint problems and optimization process is conveniently easy but when it applied to multiple constraint problems the SIMP method was unable to reach a near global optima whereas GA could reach the near global optima. Table 5 shows the comparison of computing effort of both the approaches in term of equivalent FE evaluations. It can be seen that ration of computing effort of GA to SIMP is well with in 2 which shows that GA takes twice as time taken by SIMP method.

\section{Conclusions}

Among the two algorithms which are compared in the present investigation it is found that the Three Phase Hybrid GA is robust and converges to better optimum in comparison with SIMP solution for these complex multiple constraint problems. In terms of computational effort (CE) Hybrid GA requires at least 15-20 times of CPU time in comparison with SIMP for shell problems with three compliance constraints. Further studies on both the methods are in progress for possible inclusion in any general purpose Design Optimization software.

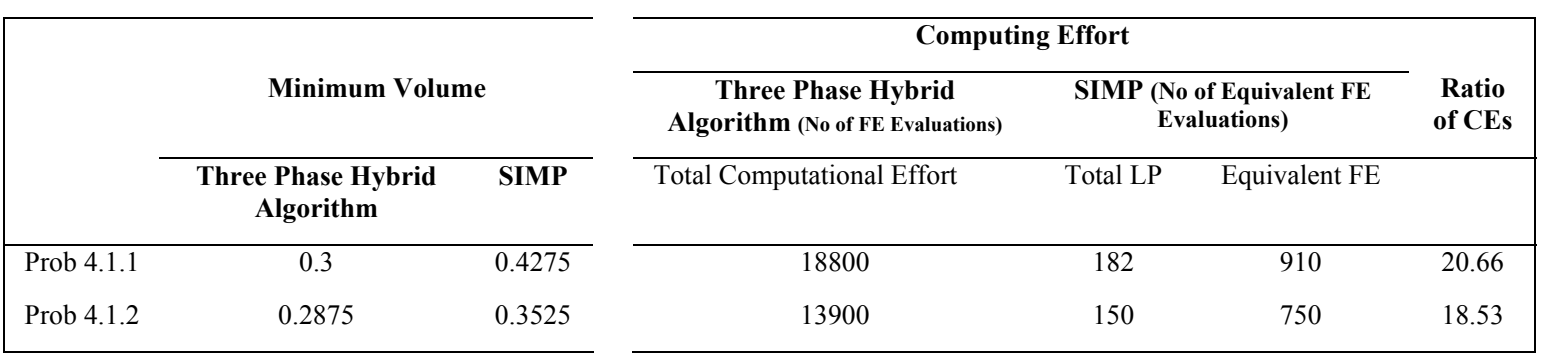

Table 4: Comparison of the methods for optimum solution and computational effort

\section{References}

[1] Maute K and Ramm E. Adaptive Topology Optimization of Shell Structures. AIAA Journal 1997; 35: 1767- 1773 .

[2] Afonso, SMB, Sienz, J. and Belblidia F. Structural optimization strategies for simple and integrally stiffened plates and shells. International Journal for Computer Aided Engineering and Software 2005; 22 (4) 429-452.

[3] Balamurugan R. Topology and shape optimization of structures. Ph.D. Thesis, I.I.T, Delhi, India, 2007.

[4] Balamurugan R, Ramakrishnan CV and Nidur Singh. Performance evaluation of Two Stage Genetic Algorithm for Topology Optimization for accelerated Convergence. Paper Accepted in Int. Journal of Applied Soft Computing, 2007

[5] Ramakrishnan CV and Nidur Singh. A Three Phase Hybrid GA Approach for Structural Topology Optimization using Genetic Algorithm and Sequential Integer Programming. ISEC 2007; Melbourne Australia. 